DOI 10.4171/JEMS/245

Enrique Zuazua

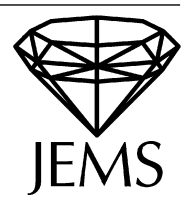

\title{
Switching control
}

Received July 25, 2008 and in revised form October 5, 2008

\begin{abstract}
We analyze the problem of switching controls for control systems endowed with different actuators. The goal is to control the dynamics of the system by switching from an actuator to another in a systematic way so that, at each instant of time, only one actuator is active. We first address a finite-dimensional model and show that, under suitable rank conditions, switching control strategies exist and can be built in a systematic way. To do this we introduce a new variational principle building a new functional based on the adjoint system whose minimizers yield the switching controls. When the above rank condition fails, the same variational strategy applies but the controls obtained this way fail to be of switching form since they may be, for some instants of time, convex combinations of both controllers. We then address the same issue for the 1-d heat equation endowed with two pointwise controls. We show that, due to the time analyticity of solutions, under suitable conditions on the location of the controllers, switching control strategies exist. We also show that the controls we obtain are optimal in the sense that, for instance, for two scalar valued controls, they are of minimal $L^{2}\left(0, T ; \mathbb{R}^{2}\right)$-norm, the space $\mathbb{R}^{2}$ being endowed with the $\ell^{1}$-norm. We also discuss some possible extensions to multi-dimensional heat equations which require a preliminary analysis of generic properties of the spectrum that, as far as we know, are not yet well understood.
\end{abstract}

Keywords. Switching control, variational approach, rank conditions, finite-dimensional system, heat equation, genericity

\section{Introduction}

Control systems in real applications are often endowed with several actuators. It is then desirable to design switching control strategies guaranteeing that, at each instant of time, only one control is activated.

In this article we develop a first analysis of this problem of switching controls addressing some model cases, although the ideas and methods we use can be greatly generalized.

We first consider the case of linear finite-dimensional systems. We show that, under suitable rank conditions, switching controllers exist. Furthermore we introduce a variational principle for the solutions of the adjoint system and show that, under those

E. Zuazua: IKERBASQUE, Basque Foundation for Science, E-48011 Bilbao, Basque Country, Spain, and Basque Center for Applied Mathematics (BCAM), Bizkaia Technology Park, Building 500, E-48160 Derio, Basque Country, Spain; e-mail: zuazua@bcamath.org

Mathematics Subject Classification (2010): 93B05, 93C20 
rank conditions, minimizers exist and lead naturally to switching controls, although their uniqueness may not be guaranteed due to the lack of strict convexity. We also show that the controls obtained in this way, in addition to being of switching form, fulfill a certain minimality condition.

The same variational strategy may be applied in the absence of the rank conditions above, provided the system is controllable by means of the simultaneous use of both controllers. However, the controls obtained this way in this case fail to be of switching form since they may be, for some instants of time, convex combinations of both controllers.

We then analyze how these methods can be adapted to some infinite-dimensional systems for which solutions are known to be time analytic. As an example of application we consider the 1-d heat equation with two pointwise controllers. Under suitable conditions on the placement of actuators we show that our approach allows building switching controls. The techniques we use in this case are inspired by those developed in [1] for oscillating controllers.

We then analyze the multi-dimensional heat equation with two controls located on two different open subsets, for which the methods we develop apply as well. However, in order for the method we propose to be effective and yield controls of genuinely switching form, a spectral non-degeneracy condition has to be fulfilled. For this spectral condition to be true the spectrum of the Laplacian must be simple, but this last generic property fails to be sufficient. As we shall see, actually, a number of interesting open problems arise on these generic spectral properties.

The method we propose fails when the evolution problem under consideration is lacking time analyticity. In that case, although our method leads to effective control strategies, the switching property may fail. This issue has recently been further investigated by M. Gugat [5] for the 1-d wave equation where the use of d'Alembert's formula allows giving a sufficient condition for the existence of switching controls. In [5] it has also been shown that the switching controls of minimal norm, when they exist, are not necessarily unique. In those cases our variational approach allows building distinguished switching controls of minimal norm.

To some extent the main ideas we develop here are similar to those employed to analyze the existence of bang-bang controls ([3]). In the latter context the switching requirement is imposed on the extremal values of the same controller, while here switching refers to a change of the actuator that is activated at each time instant ([9], [6], [19]). As we shall see, the combination of these ideas allows also building switching bang-bang controls.

Switching controllers arise in many fields of applications (see the survey article [16]). Switching may also refer to the possibility of the state equation itself to change from one configuration to another at some time instants (see [7] for an application to transport on networks). This issue is not analyzed in this paper. It should be emphasized that our analysis only refers to the case where the state equation is given a priori and where only the controllers are allowed to switch. In this setting the problem can be dealt with the variants of the existing linear theory we develop here. Dealing with the case where the state equation itself may switch requires further important developments. 


\section{Finite-dimensional linear systems}

\subsection{The main result}

Consider the finite-dimensional linear control system

$$
\left\{\begin{array}{l}
x^{\prime}(t)=A x(t)+u_{1}(t) b_{1}+u_{2}(t) b_{2}, \\
x(0)=x^{0}
\end{array}\right.
$$

In 2.1 the (column) vector valued function $x(t)=\left(x_{1}(t), \ldots, x_{N}(t)\right) \in \mathbb{R}^{N}$ is the state of the system, $A$ is an $N \times N$ matrix, $u_{1}=u_{1}(t)$ and $u_{2}=u_{2}(t)$ are two scalar controls and $b_{1}, b_{2}$ are given (column) control vectors in $\mathbb{R}^{N}$.

We address the problem of controllability. Given a control time $T>0$ and a final target $x^{1} \in \mathbb{R}^{N}$ we look for control pairs $\left(u_{1}, u_{2}\right)$ such that the solution of 2.1 satisfies

$$
x(T)=x^{1}
$$

In the absence of constraints on the controls, controllable systems can be fully characterized in algebraic terms. Indeed, if $B=\left(b_{1}, b_{2}\right)$ is the $N \times 2$ matrix containing the two column vectors $b_{1}$ and $b_{2}$, the Kalman rank condition which is necessary and sufficient for the controllability of 2.1) is as follows:

$$
\operatorname{rank}\left[B, A B, \ldots, A^{N-1} B\right]=N .
$$

Here we are interested in analyzing the existence of switching controls $u_{1}=u_{1}(t)$ and $u_{2}=u_{2}(t)$ such that

$$
u_{1}(t) u_{2}(t)=0, \quad \text { a.e. } t \in(0, T)
$$

Condition 2.4 ensures that, at each instant of time, at most one of the two controllers is active. Of course, when one single control $u_{1}$ or $u_{2}$ suffices for controlling the system, condition 2.4 may be trivially achieved by taking simply the other one to vanish. But this would require the rank condition $(2.3)$ to be satisfied on replacing $B$ by $b_{1}$ or $b_{2}$, and this is, obviously, not guaranteed under assumption $(2.3)$ above for $B=\left(b_{1}, b_{2}\right)$. Despite this ${ }^{1}$ as we shall see, in the present finite-dimensional setting, this weaker rank condition suffices to guarantee the existence of these switching controls. To be more precise, as we shall see, one can set a priori a splitting strategy by choosing a partition of the time interval and imposing the controls to alternate following that partition. Controllability then holds by means of switching strategies given a priori, depending on the partition of the time interval $[0, T]$ one chooses.

Here we are interested in developing a systematic method allowing one to get switching controls without imposing a priori an artificial partition of the time interval. The controls we shall build are characterized through a variational principle which guarantees that

\footnotetext{
1 We thank Th. Seidman for this interesting observation.
} 
they are optimal in the sense that they are of minimal norm in $L^{2}\left(0, T ; \mathbb{R}^{2}\right)$, the space $\mathbb{R}^{2}$ being endowed with the $\ell^{1}$-norm, i.e. with respect to the norm

$$
\left\|\left(u_{1}, u_{2}\right)\right\|_{L^{2}\left(0, T ; \ell^{1}\right)}=\left[\int_{0}^{T}\left(\left|\tilde{u}_{1}\right|+\left|\tilde{u}_{2}\right|\right)^{2} d t\right]^{1 / 2} .
$$

The problem is that the condition that the pair $(A, B)$ satisfies the Kalman rank condition does not suffice. Actually one needs that both pairs $\left(A, b_{1}+b_{2}\right)$ and $\left(A, b_{1}-b_{2}\right)$ satisfy the Kalman rank condition, which is a stronger condition than simply requiring it for the pair $(A, B)$, of course. One can slightly modify the argument by weighting differently the two controllers so that the sufficient condition for switching control becomes that both pairs $\left(A, b_{1}+\alpha b_{2}\right)$ and $\left(A, b_{1}-\alpha b_{2}\right)$ satisfy the Kalman rank condition for some $\alpha>0$.

Our analysis of this problem is based on minimizing suitable functionals associated to the adjoint system

$$
\left\{\begin{array}{l}
-\varphi^{\prime}(t)=A^{*} \varphi(t), \quad t \in(0, T) \\
\varphi(T)=\varphi^{0}
\end{array}\right.
$$

The novelty of the approach we propose relies on the new class of functionals we introduce.

Let us first recall some elements of the existing theory.

The classical theory of controllability (see [19]) guarantees that, under the condition 2.3, the controls $\left(u_{1}, u_{2}\right)$ may be built by minimizing the functional

$$
J\left(\varphi^{0}\right)=\frac{1}{2} \int_{0}^{T}\left[\left|b_{1} \cdot \varphi(t)\right|^{2}+\left|b_{2} \cdot \varphi(t)\right|^{2}\right] d t-x^{1} \cdot \varphi^{0}+x^{0} \cdot \varphi(0) .
$$

Here and below, we denote by $\cdot$ the scalar product in the euclidean space $\mathbb{R}^{N}$.

The functional $J: \mathbb{R}^{N} \rightarrow \mathbb{R}$ is trivially continuous and convex. On the other hand, the coercivity of $J$ is equivalent to the rank condition 2.3$)$ for $B=\left(b_{1}, b_{2}\right)$. To be more precise, as shown in [18], for instance, this rank condition is equivalent to the following unique-continuation property:

$$
\left(b_{1} \cdot \varphi(t)=b_{2} \cdot \varphi(t)=0, \forall t \in[0, T]\right) \Rightarrow \varphi \equiv 0,
$$

which is itself equivalent to the coercivity of $J$.

Under the rank condition 2.3 , the functional $J$ achieves its minimum at a single $\check{\varphi}^{0}$, which corresponds to a unique solution $\check{\varphi}$ of the adjoint system 2.6. yielding the controls

$$
u_{1}(t)=b_{1} \cdot \check{\varphi}(t), \quad u_{2}(t)=b_{2} \cdot \check{\varphi}(t)
$$

which fulfill the final requirement 2.2 , and turn out to be of minimal $L^{2}\left(0, T ; \mathbb{R}^{2}\right)$-norm, the image space $\mathbb{R}^{2}$ being endowed with the standard euclidean norm.

However, the switching condition (2.4) fails in general for this choice of controls. Indeed, in view of the structure of the controls we obtain in 2.80 it is easy to see that, generically, each of the controls vanishes only at a finite number of time instances. 
Remark 2.1. As mentioned above, the same argument allows considering, for a given partition $\tau=\left\{t_{0}=0<t_{1}<\cdots<t_{2 N}=T\right\}$ of the time interval $(0, T)$, a functional of the form

$$
\begin{aligned}
J_{\tau}\left(\varphi^{0}\right)= & \frac{1}{2} \sum_{j=0}^{N-1} \int_{t_{2 j}}^{t_{2 j+1}}\left|b_{1} \cdot \varphi(t)\right|^{2} d t \\
& +\frac{1}{2} \sum_{j=0}^{N-1} \int_{t_{2 j+1}}^{t_{2 j+2}}\left|b_{2} \cdot \varphi(t)\right|^{2} d t-x^{1} \cdot \varphi^{0}+x^{0} \cdot \varphi(0) .
\end{aligned}
$$

Under the same rank condition this functional is coercive too. In fact, in view of the time analyticity of solutions, the above unique continuation property implies the apparently stronger one:

$$
\begin{array}{r}
{\left[b_{1} \cdot \varphi(t)=0, t \in\left(t_{2 j}, t_{2 j+1}\right) ; b_{2} \cdot \varphi(t)=0, t \in\left(t_{2 j+1}, t_{2 j+2}\right), j=0, \ldots, N-1\right]} \\
\Rightarrow \varphi \equiv 0,
\end{array}
$$

and this suffices to show the coercivity of $J_{\tau}$. Thus, $J_{\tau}$ has a unique minimizer $\check{\varphi}$ and this yields the controls

$$
\begin{array}{ll}
u_{1}(t)=b_{1} \cdot \check{\varphi}(t), \quad t \in\left(t_{2 j}, t_{2 j+1}\right) ; \\
u_{2}(t)=b_{2} \cdot \check{\varphi}(t), \quad t \in\left(t_{2 j+1}, t_{2 j+2}\right), \quad j=0, \ldots, N-1,
\end{array}
$$

which are obviously of switching form.

Of course a different ordering of both controllers in the subintervals is also possible and yields the same result.

Note however that this argument requires introducing a priori a partition of the time interval and that the controls one obtains depend on it.

The key observation of this article is that, under further rank conditions, the following functional, which is a variant of the one in 2.7, with the same coercivity properties, allows building switching controllers:

$$
J_{S}\left(\varphi^{0}\right)=\frac{1}{2} \int_{0}^{T} \max \left(\left|b_{1} \cdot \varphi(t)\right|^{2},\left|b_{2} \cdot \varphi(t)\right|^{2}\right) d t-x^{1} \cdot \varphi^{0}+x^{0} \cdot \varphi(0) .
$$

The following holds:

Theorem 2.1. Assume that the pairs $\left(A, b_{2}-b_{1}\right)$ and $\left(A, b_{2}+b_{1}\right)$ satisfy the rank condition 2.3. Then, for all $T>0$, the functional $J_{S}$ in 2.10) achieves at least one minimum at some minimizer $\tilde{\varphi}^{0}$. Furthermore, the switching controllers

$$
\begin{cases}u_{1}(t)=\tilde{\varphi}(t) \cdot b_{1} & \text { when }\left|\tilde{\varphi}(t) \cdot b_{1}\right|>\left|\tilde{\varphi}(t) \cdot b_{2}\right|, \\ u_{2}(t)=\tilde{\varphi}(t) \cdot b_{2} & \text { when }\left|\tilde{\varphi}(t) \cdot b_{2}\right|>\left|\tilde{\varphi}(t) \cdot b_{1}\right|,\end{cases}
$$

where $\tilde{\varphi}$ is the solution of (2.6) with datum $\tilde{\varphi}^{0}$ at time $t=T$, are such that the solution of 2.1) satisfies the final requirement 2.2. 
Remark 2.2. - It is interesting to observe that we impose the condition that the pairs $\left(A, b_{2} \pm b_{1}\right)$ satisfy the Kalman rank condition. This is a necessary and sufficient condition for the controllability of the systems

$$
x^{\prime}+A x=\left(b_{2} \pm b_{1}\right) u(t)
$$

The fact that these systems are controllable implies that system 2.1) is controllable as well and this is equivalent to the fact that the pair $(A, B)$, with $B=\left(b_{1}, b_{2}\right)$, satisfies the same condition. But the latter does not suffice to ensure that the controls obtained by minimizing $J_{s}$ are of switching form. In fact, as we shall see below, without extra rank conditions the controls obtained by minimizing $J_{S}$ may be convex combinations of both controllers $b_{1}, b_{2}$ at some instances.

- The rank conditions on the pairs $\left(A, b_{2} \pm b_{1}\right)$ are needed to ensure that the switching controls in 2.11 are well defined, i.e. to guarantee that the set

$$
\left\{t \in(0, T):\left|\varphi(t) \cdot b_{1}\right|=\left|\varphi(t) \cdot b_{2}\right|\right\}
$$

is of zero measure, which ensures that the controls in 2.11 are genuinely of switching form. Indeed, the choice (2.11) of the controls $u_{1}$ and $u_{2}$ guarantees the switching structure if and only if the set in 2.13 is of null measure.

- Similar conditions on the pairs $\left(A, b_{1} \pm b_{2}\right)$ appear when dealing with minimal time controls under polyhedral constraints (see, for instance, Corollary 1 on p. 133 of [9]).

Proof of Theorem 2.1. Without loss of generality we can assume that $x^{1} \neq e^{A T} x^{0}$. Indeed, when $x^{1}=e^{A T} x^{0}$ the null controls $u_{1} \equiv u_{2} \equiv 0$ suffice to drive the initial datum $x^{0}$ to the final one $x^{1}$, and they satisfy the switching condition 2.4 . Thus, in what follows, we assume that $x^{0}$ and $x^{1}$ are such that $x^{1} \neq e^{A T} x^{0}$.

The functional $J_{S}: \mathbb{R}^{N} \rightarrow \mathbb{R}$ in 2.10 is clearly continuous and convex. Therefore, the existence of a minimizer will be guaranteed as soon as the coercivity of $J_{S}$ holds. Under the assumptions of Theorem 2.1 the functional $J_{s}$ is coercive, i.e.,

$$
\lim _{\left\|\varphi^{0}\right\| \rightarrow \infty} \frac{J_{s}\left(\varphi^{0}\right)}{\left\|\varphi^{0}\right\|}=\infty .
$$

To see this it is sufficient to show that there exists a positive constant $C>0$ such that

$$
\left\|\varphi^{0}\right\|^{2} \leq C \int_{0}^{T} \max \left\{\left|\varphi(t) \cdot b_{1}\right|^{2},\left|\varphi(t) \cdot b_{2}\right|^{2}\right\} d t .
$$

This is immediate if we take into account that

$$
\left|\varphi(t) \cdot b_{1}\right|^{2}+\left|\varphi(t) \cdot b_{2}\right|^{2} \leq 2 \max \left[\left|\varphi(t) \cdot b_{1}\right|^{2},\left|\varphi(t) \cdot b_{2}\right|^{2}\right]
$$

and that the inequality

$$
\left\|\varphi^{0}\right\|^{2} \leq C \int_{0}^{T}\left[\left|\varphi(t) \cdot b_{1}\right|^{2}+\left|\varphi(t) \cdot b_{2}\right|^{2}\right] d t
$$

holds as well, since the pair $(A, B)$ satisfies the rank condition (see [18]). 
Let $\tilde{\varphi}^{0}$ be a minimizer of $J_{s}$ and $\tilde{\varphi}$ the corresponding solution of 2.6), and let us write the Euler-Lagrange equations for the minimization of $J_{s}$.

At this point the following property plays a key role: under the assumptions of Theorem 2.1 the set

$$
I=\left\{t \in(0, T):\left|\tilde{\varphi} \cdot b_{1}\right|=\left|\tilde{\varphi} \cdot b_{2}\right|\right\}
$$

is of null measure. Indeed, the set $I$ is in fact the union of the two subsets

$$
I_{ \pm}=\left\{t \in(0, T): \tilde{\varphi}(t) \cdot b_{1}= \pm \tilde{\varphi}(t) \cdot b_{2}\right\},
$$

both of null measure. To see this assume, for instance, that the set $I_{+}=\{t \in(0, T)$ : $\left.\tilde{\varphi}(t) \cdot\left(b_{1}-b_{2}\right)=0\right\}$ is of positive measure. Then the time analyticity of $\tilde{\varphi} \cdot\left(b_{1}-b_{2}\right)$ implies that $I_{+}=(0, T)$. Accordingly $\tilde{\varphi} \cdot\left(b_{1}-b_{2}\right) \equiv 0$, and consequently, taking into account that the pair $\left(A, b_{1}-b_{2}\right)$ satisfies the Kalman rank condition, this implies that $\tilde{\varphi} \equiv 0$. This would imply that the minimum of $J_{s}$ is achieved at $\tilde{\varphi}^{0} \equiv 0$, which would mean that

$$
J_{s}\left(\varphi^{0}\right)=\frac{1}{2} \int_{0}^{T} \max \left(\left|\varphi(t) \cdot b_{1}\right|^{2},\left|\varphi(t) \cdot b_{2}\right|^{2}\right) d t-x^{1} \cdot \varphi_{0}+x^{0} \cdot \varphi(0) \geq 0, \quad \forall \varphi^{0} \in \mathbb{R}^{N} .
$$

Taking into account that

$$
\max \left(\left|\varphi(t) \cdot b_{1}\right|^{2},\left|\varphi(t) \cdot b_{2}\right|^{2}\right) \leq\left|\varphi(t) \cdot b_{1}\right|^{2}+\left|\varphi(t) \cdot b_{2}\right|^{2}
$$

this would imply that

$$
J\left(\varphi^{0}\right) \geq 0, \quad \forall \varphi^{0} \in \mathbb{R}^{N},
$$

as well, and the minimizer of $J$ as in (2.7) would also be the trivial state. This corresponds to the case that the system (2.1) reaches the target 2.2) by means of the trivial controls $u_{1} \equiv u_{2} \equiv 0$. This can only happen in the case where $x^{1}=e^{A T} x^{0}$, which has been excluded.

We are now in a position to derive the Euler-Lagrange equations.

For any $\psi^{0} \in \mathbb{R}^{N}$ we have

$$
J_{S}\left(\tilde{\varphi}^{0}\right) \leq J_{S}\left(\tilde{\varphi}^{0}+h \psi^{0}\right), \quad \forall h .
$$

Therefore

$$
\begin{array}{r}
x^{1} \cdot \psi^{0}-x^{0} \cdot \psi(0) \leq \lim _{h \rightarrow 0^{+}} \frac{1}{2 h} \int_{0}^{T}\left[\max \left(\left|(\tilde{\varphi}+h \psi) \cdot b_{1}\right|^{2},\left|(\tilde{\varphi}+h \psi) \cdot b_{2}\right|^{2}\right)\right. \\
\left.-\max \left(\left|\tilde{\varphi} \cdot b_{1}\right|^{2},\left|\tilde{\varphi} \cdot b_{2}\right|^{2}\right)\right] d t,
\end{array}
$$

where $\psi=\psi(t)$ stands for the solution of the adjoint system 2.6 with $\psi^{0}$ as datum at time $t=T$.

We claim that the limit on the right hand side of 2.16 coincides with

$$
\int_{S_{1}} \tilde{\varphi} \cdot b_{1} \psi \cdot b_{1} d t+\int_{S_{2}} \tilde{\varphi} \cdot b_{2} \psi \cdot b_{2} d t
$$


where

$$
\left\{\begin{array}{l}
S_{1}=\left\{t \in(0, T):\left|\tilde{\varphi}(t) \cdot b_{1}\right|>\left|\tilde{\varphi}(t) \cdot b_{2}\right|\right\}, \\
S_{2}=\left\{t \in(0, T):\left|\tilde{\varphi}(t) \cdot b_{1}\right|<\left|\tilde{\varphi}(t) \cdot b_{2}\right|\right\} .
\end{array}\right.
$$

At this point it is essential that the set $I$ as in (2.14) is of null measure.

To see this we first observe that, pointwise a.e. in $(0, T)$, the following holds:

$$
\begin{aligned}
\frac{1}{2 h}\left[\max \left(\left|(\tilde{\varphi}+h \psi) \cdot b_{1}\right|^{2},\left|(\tilde{\varphi}+h \psi) \cdot b_{2}\right|^{2}\right)-\right. & \left.\max \left(\left|\tilde{\varphi} \cdot b_{1}\right|^{2},\left|\tilde{\varphi} \cdot b_{2}\right|^{2}\right)\right] \\
& \rightarrow \begin{cases}\tilde{\varphi} \cdot b_{1} \psi \cdot b_{1}, & \text { a.e. } t \in S_{1}, \\
\tilde{\varphi} \cdot b_{2} \psi \cdot b_{2}, & \text { a.e. } t \in S_{2},\end{cases}
\end{aligned}
$$

as $h \rightarrow 0$. This is an immediate consequence of the fact that the sets $I_{ \pm}$in 2.15 are of zero measure and that, within $S_{1}$ (resp. $S_{2}$ ), for $h$ sufficiently small (depending on $t$ ), $\max \left(\left|(\tilde{\varphi}+h \psi) \cdot b_{1}\right|^{2},\left|(\tilde{\varphi}+h \psi) \cdot b_{2}\right|^{2}\right)$ is simply $\left|(\tilde{\varphi}+h \psi) \cdot b_{1}\right|^{2}\left(\operatorname{resp} .\left|(\tilde{\varphi}+h \psi) \cdot b_{2}\right|^{2}\right)$.

To get the limit 2.17$)$ it is then sufficient to apply the dominated convergence theorem. To do that it suffices to show that

$$
\begin{aligned}
\frac{1}{h} \mid\left(\left|(\tilde{\varphi}(t)+h \psi(t)) \cdot b_{1}\right|^{2},\left|(\tilde{\varphi}(t)+h \psi(t)) \cdot b_{2}\right|^{2}\right)- & \max \left(\left|\tilde{\varphi}(t) \cdot b_{1}\right|^{2},\left|\tilde{\varphi}(t) \cdot b_{2}\right|^{2}\right) \mid \\
& \leq C(t), \quad \text { a.e. } t \in(0, T),
\end{aligned}
$$

with $C(t) \in L^{1}(0, T)$ depending on $\psi$, for all $h>0$ small enough.

The only difficulty in proving this kind of uniform bound arises on the set where the two maxima are not taken over the same component. Indeed, when both maxima are taken over the same component, for instance, if

$$
\max \left(\left|(\tilde{\varphi}(t)+h \psi(t)) \cdot b_{1}\right|^{2},\left|(\tilde{\varphi}(t)+h \psi(t)) \cdot b_{2}\right|^{2}\right)=\left|(\tilde{\varphi}(t)+h \psi(t)) \cdot b_{1}\right|^{2}
$$

and

$$
\max \left(\left|\tilde{\varphi}(t) \cdot b_{1}\right|^{2},\left|\tilde{\varphi}(t) \cdot b_{2}\right|^{2}\right)=\left|\tilde{\varphi}(t) \cdot b_{1}\right|^{2},
$$

then the quotient in 2.19$)$ can be bounded above by $2\left|\tilde{\varphi}(t) \cdot b_{1}\right|\left|\psi(t) \cdot b_{1}\right|$, which is in $L^{1}(0, T)$ since both $\tilde{\varphi}$ and $\psi$ belong to $L^{2}(0, T)$. A similar argument can be applied when the maxima are taken over the second components.

Let us then consider the remaining case where, for instance,

$$
\max \left(\left|(\tilde{\varphi}(t)+h \psi(t)) \cdot b_{1}\right|^{2},\left|(\tilde{\varphi}(t)+h \psi(t)) \cdot b_{2}\right|^{2}\right)=\left|(\tilde{\varphi}(t)+h \psi(t)) \cdot b_{1}\right|^{2}
$$

but

$$
\max \left(\left|\tilde{\varphi}(t) \cdot b_{1}\right|^{2},\left|\tilde{\varphi}(t) \cdot b_{2}\right|^{2}\right)=\left|\tilde{\varphi}(t) \cdot b_{2}\right|^{2} .
$$

In that case the quotient in 2.19 coincides with

$$
\begin{aligned}
& \frac{1}{h}\left[\left|(\tilde{\varphi}(t)+h \psi(t)) \cdot b_{1}\right|^{2}-\left|\tilde{\varphi}(t) \cdot b_{2}\right|^{2}\right] \\
& \quad=\left[\left|(\tilde{\varphi}(t)+h \psi(t)) \cdot b_{1}\right|+\left|\tilde{\varphi}(t) \cdot b_{2}\right|\right] \frac{1}{h}\left[\left|(\tilde{\varphi}(t)+h \psi(t)) \cdot b_{1}\right|-\left|\tilde{\varphi}(t) \cdot b_{2}\right|\right] .
\end{aligned}
$$


It is then sufficient to get an upper bound on

$$
\frac{1}{h}||(\tilde{\varphi}(t)+h \psi(t)) \cdot b_{1}|-| \tilde{\varphi}(t) \cdot b_{2}|| \leq\left|\psi(t) \cdot b_{1}\right|+\left|\frac{\left|\tilde{\varphi}(t) \cdot b_{1}\right|-\left|\tilde{\varphi}(t) \cdot b_{2}\right|}{h}\right| .
$$

To do this the only difficulty is to get an upper bound on

$$
\left|\frac{\left|\tilde{\varphi}(t) \cdot b_{1}\right|-\left|\tilde{\varphi}(t) \cdot b_{2}\right|}{h}\right| \text {. }
$$

But, obviously, for 2.20) and (2.21) to hold we need that

$$
|h|\left|\psi(t) \cdot b_{1}\right|+|h|\left|\psi(t) \cdot b_{2}\right|>\left|\tilde{\varphi}(t) \cdot b_{2}\right|-\left|\tilde{\varphi}(t) \cdot b_{1}\right|=|| \tilde{\varphi}(t) \cdot b_{2}|-| \tilde{\varphi}(t) \cdot b_{1}||,
$$

which also guarantees the uniform boundedness of (2.23).

As a consequence of this analysis, the Euler-Lagrange equations associated to the minimization of $J_{S}$ take the form

$$
\int_{S_{1}} \tilde{\varphi}(t) \cdot b_{1} \psi(t) \cdot b_{1} d t+\int_{S_{2}} \tilde{\varphi}(t) \cdot b_{2} \psi(t) \cdot b_{2} d t-x^{1} \cdot \psi^{0}+x^{0} \cdot \psi(0)=0
$$

for all $\psi^{0} \in \mathbb{R}^{N}$. In view of 2.24) we conclude that the switching controllers

$$
u_{1}(t)=\tilde{\varphi}(t) \cdot b_{1} 1_{S_{1}}(t), \quad u_{2}(t)=\tilde{\varphi}(t) \cdot b_{2} 1_{S_{2}}(t),
$$

where $1_{S_{1}}$ and $1_{S_{2}}$ stand for the characteristic functions of the sets $S_{1}$ and $S_{2}$ in 2.18 , are such that the switching condition (2.4) holds and the solution of 2.1) satisfies the final requirement 2.2.

This concludes the proof of Theorem 2.1

\subsection{Some extensions}

The main result in Theorem 2.1 can be extended in various ways. We comment here on some possibilities.

Other switching strategies. Similar arguments can be applied for variants of the functional $J_{S}$ of the form

$$
J_{s}^{\alpha}\left(\varphi^{0}\right)=\frac{1}{2} \int_{0}^{T} \max \left(\left|b_{1} \cdot \varphi(t)\right|^{2},\left|\alpha b_{2} \cdot \varphi(t)\right|^{2}\right) d t-x^{1} \cdot \varphi^{0}+x^{0} \cdot \varphi(0)
$$

with $\alpha>0$.

In this case the sufficient condition for the controls obtained by minimizing this functional to be of switching form is that the pairs $\left(A, b_{1}+\alpha b_{2}\right)$ and $\left(A, b_{1}-\alpha b_{2}\right)$ satisfy the Kalman rank condition. As a consequence, in particular, we see that, under the sole condition that the pair $\left(A, b_{1}\right)$ satisfies the Kalman rank condition (which ensures that the system is controllable using only the controller $u_{1}$ ), this switching strategy applies for some $\alpha>0$ since, for $\alpha$ small enough, by continuity, the pairs $\left(A, b_{1} \pm \alpha b_{2}\right)$ satisfy the Kalman rank condition as well. The same argument applies when the Kalman rank 
condition is satisfied by $\left(A, b_{2}\right)$. It is then sufficient to minimize the functional $J_{s}^{\alpha}$ for $\alpha$ large enough.

In this case the switching controls obtained are of the form

$$
\begin{cases}u_{1}(t)=\tilde{\varphi}(t) \cdot b_{1} & \text { when }\left|\tilde{\varphi}(t) \cdot b_{1}\right|>\left|\alpha \tilde{\varphi}(t) \cdot b_{2}\right| \\ u_{2}(t)=\alpha^{2} \tilde{\varphi}(t) \cdot b_{2} & \text { when }\left|\tilde{\varphi}(t) \cdot b_{2}\right|>\left|\alpha \tilde{\varphi}(t) \cdot b_{1}\right| .\end{cases}
$$

Time dependent switching strategies. The same functional as above can also be considered with $\alpha=\alpha(t)$, a time dependent function:

$$
J_{s}^{\alpha}\left(\varphi^{0}\right)=\frac{1}{2} \int_{0}^{T} \max \left(\left|b_{1} \cdot \varphi(t)\right|^{2},\left|\alpha(t) b_{2} \cdot \varphi(t)\right|^{2}\right) d t-x^{1} \cdot \varphi^{0}+x^{0} \cdot \varphi(0),
$$

with $\alpha(t)>0$ for a.e. $t \in[0, T]$.

To show that the minimizer of this functional yields a control of switching form we have to show that there is no non-trivial solution satisfying

$$
b_{1} \cdot \varphi(t)= \pm \alpha(t) b_{2} \cdot \varphi(t)
$$

on a set of positive measure.

At this point we can use the arguments in [1].

Assume that $\alpha=\alpha(t)$, which, a priori, is defined on the time interval [0,T], can be extended analytically to the whole real line. Then, using the time analyticity of $\varphi(t)$, we can deduce that if either $b_{1} \cdot \varphi(t)=\alpha(t) b_{2} \cdot \varphi(t)$ or $b_{1} \cdot \varphi(t)=-\alpha(t) b_{2} \cdot \varphi(t)$ vanishes on a set of positive measure, then they vanish everywhere.

Let us assume, for instance, that $b_{1} \cdot \varphi(t)-\alpha(t) b_{2} \cdot \varphi(t) \equiv 0$ for all $t$.

We claim that, in order to guarantee that this condition implies that $\varphi \equiv 0$, as desired, it is sufficient that the pair $\left(A, b_{1}-\alpha_{\infty} b_{2}\right)$ satisfies the Kalman rank condition for some $\alpha_{\infty}$ in the set of accumulation points of $\{\alpha(t)\}$ at infinity.

We decompose the solution $\varphi$ in the basis of eigenvectors of the matrix $A$ :

$$
\varphi(t)=\sum_{\lambda} a_{\lambda} e^{\lambda t} w_{\lambda}
$$

Let $\Lambda$ be the eigenvalue with the greatest real part and assume it is unique. Then, if $b_{1} \cdot \varphi(t)-\alpha(t) b_{2} \cdot \varphi(t) \equiv 0$ we also have

$$
a_{\Lambda}\left(b_{1} \cdot w_{\Lambda}-\alpha(t) b_{2} \cdot w_{\Lambda}\right)+\sum_{\lambda \neq \Lambda} a_{\lambda} e^{(\lambda-\Lambda) t}\left(b_{1} \cdot w_{\lambda}-\alpha(t) b_{2} \cdot w_{\lambda}\right) \equiv 0 .
$$

Passing to the limit as $t \rightarrow \infty$ we deduce that $a_{\Lambda}\left(b_{1} \cdot w_{\Lambda}-\alpha_{\infty} b_{2} \cdot w_{\Lambda}\right)=0$ for all $\alpha_{\infty}$ in the set of accumulation points of $\{\alpha(t)\}$ as $t \rightarrow \infty$. This, together with the fact that the pair $\left(A, b_{1}-\alpha_{\infty} b_{2}\right)$ satisfies the Kalman rank condition, guarantees that $a_{\Lambda}=0$. By induction this argument allows showing that $a_{\lambda}=0$ for all $\lambda$ provided the real parts of all the eigenvalues of $A$ are distinct.

In case there are several eigenvalues with equal real parts the argument above does not work. Indeed, if there are, for instance, several eigenvalues with the same real part as 
that of $\Lambda$, the leading term in the expansion above, instead of being time independent, is a finite combination of complex exponentials:

$$
\sum_{\operatorname{Re}(\lambda)=\operatorname{Re}(\Lambda)} a_{\lambda} e^{i \operatorname{Im}(\lambda-\Lambda) t}\left(b_{1} \cdot w_{\lambda}-\alpha(t) b_{2} \cdot w_{\lambda}\right)
$$

which tends to zero as $t \rightarrow \infty$. In this case passing to the limit $t \rightarrow \infty$ along sequences so that $\alpha(t)$ (resp. $e^{i \operatorname{Im}(\lambda-\Lambda) t}$ ) accumulates around $\alpha_{\infty}$ (resp. $c_{\lambda, \infty}$ ), we deduce that

$$
\sum_{\operatorname{Re}(\lambda)=\operatorname{Re}(\Lambda)} a_{\lambda} c_{\lambda, \infty}\left(b_{1} \cdot w_{\lambda}-\alpha_{\infty} b_{2} \cdot w_{\lambda}\right)=\left(b_{1}-\alpha_{\infty} b_{2}\right) \cdot \sum_{\operatorname{Re}(\lambda)=\operatorname{Re}(\Lambda)} a_{\lambda} c_{\lambda, \infty} w_{\lambda}=0 .
$$

But this is not sufficient to conclude since some non-trivial linear combinations of the eigenvectors $w_{\lambda}$ could be orthogonal to the vector $b_{1} \cdot w_{\lambda}-\alpha_{\infty} b_{2}$.

The same argument can be applied as $t \rightarrow-\infty$. This is why, under the assumption that all the real parts of the eigenvalues are distinct, it is sufficient that the Kalman rank condition is satisfied by $\left(A, b_{1}-\alpha_{\infty} b_{2}\right)$ for some $\alpha_{\infty}$ in the set of accumulation points of $\alpha(t)$ as $t \rightarrow \pm \infty$. The same can be said about the other linear combination $b_{1} \cdot \varphi(t)+$ $\alpha(t) b_{2} \cdot \varphi(t)$.

Consequently, the following holds:

Theorem 2.2. Let $\alpha=\alpha(t)$ be a real analytic function on the whole real line and let $\mathcal{A}_{ \pm}$be the set of accumulation points of $\alpha(t)$ as $t \rightarrow \pm \infty$. Assume that the real parts of the eigenvalues of $A$ are all distinct and that $\left(A, b_{1}-\alpha_{-} b_{2}\right)$ and $\left(A, b_{1}+\alpha_{+} b_{2}\right)$ satisfy the Kalman rank condition for some values of $\alpha_{ \pm}$in the sets $\mathcal{A}_{ \pm}$of accumulation points. Then minimizing the functional (2.28) yields a switching control of the form

$$
\begin{cases}u_{1}(t)=\tilde{\varphi}(t) \cdot b_{1} & \text { when }\left|\tilde{\varphi}(t) \cdot b_{1}\right|>\left|\alpha(t) \tilde{\varphi}(t) \cdot b_{2}\right| \\ u_{2}(t)=\alpha^{2}(t) \tilde{\varphi}(t) \cdot b_{2} & \text { when }\left|\tilde{\varphi}(t) \cdot b_{2}\right|>\left|\alpha(t) \tilde{\varphi}(t) \cdot b_{1}\right| .\end{cases}
$$

Remark 2.3. - As a consequence of this result, if the real parts of the eigenvalues of $A$ are all distinct and the controllers $b_{1}$ and $b_{2}$ are such that the rank condition is fulfilled for $\left(A, b_{1}-\alpha_{-} b_{2}\right)$ and $\left(A, b_{1}+\alpha_{+} b_{2}\right)$ for some values of $\alpha_{-}$and $\alpha_{+}$, regardless of whether they belong to $\mathcal{A}_{-}$or $\mathcal{A}_{+}$, then one can always find a function $\alpha=\alpha(t)$ whose set of accumulation points contains $\alpha_{ \pm}$(or even a monotonic function with those as asymptotic values) and that yields switching controls.

- We impose the assumption that the real parts of the eigenvalues are all distinct. But certainly, this assumption is not sharp. A further analysis is needed to completely identify the class of matrices $A$ for which this theorem holds.

Bang-bang switching controls. Under the assumptions of Theorem 2.1 one can slightly change the construction of the controls to build switching controllers of bang-bang form. For that it is sufficient to consider the functional

$$
J_{s b}\left(\varphi^{0}\right)=\frac{1}{2}\left[\int_{0}^{T} \max \left(\left|\varphi(t) \cdot b_{1}\right|,\left|\varphi(t) \cdot b_{2}\right|\right) d t\right]^{2}-x^{1} \cdot \varphi^{0}+x^{0} \cdot \varphi(0) .
$$


The controls obtained through the minimization of this functional are then of the form

$$
u_{1}(t)=\lambda \operatorname{sgn}\left(\tilde{\varphi}(t) \cdot b_{1}\right) 1_{S_{1}}(t), \quad u_{2}(t)=\lambda \operatorname{sgn}\left(\tilde{\varphi}(t) \cdot b_{2}\right) 1_{S_{2}}(t)
$$

where

$$
\lambda=\int_{0}^{T} \max \left(\left|\tilde{\varphi}(t) \cdot b_{1}\right|,\left|\tilde{\varphi}(t) \cdot b_{2}\right|\right) d t
$$

and the sets $S_{1}$ and $S_{2}$ are as in 2.18.

Therefore they are switching controls of bang-bang form taking the values $\pm \lambda$ when they are active.

Higher number of controllers. Similar issues can also be addressed when more than two controllers are applied. Consider, for instance, the system

$$
\left\{\begin{array}{l}
x^{\prime}=A x+\sum_{j=1}^{p} u_{j}(t) b_{j}, \quad 0<t<T, \\
x(0)=x^{0}
\end{array}\right.
$$

in which $p$ controllers enter. It is then natural to consider the functional

$$
J_{S}\left(\varphi^{0}\right)=\frac{1}{2} \int_{0}^{T} \max \left(\left|\varphi(t) \cdot b_{1}\right|^{2}, \ldots,\left|\varphi(t) \cdot b_{p}\right|^{2}\right) d t-x^{1} \cdot \varphi^{0}+x^{0} \cdot \varphi(0) .
$$

It is easy to see that it achieves its minimum under the condition that the pair $(A, B)$ satisfies the Kalman rank condition where $B=\left(b_{1}, \ldots, b_{p}\right)$.

When writing the Euler-Lagrange equations for $J_{s}$, to guarantee that the controllers obtained in this way are of switching form, one has to make sure that the sets

$$
I_{\ell m}=\left\{t \in(0, T):\left|\varphi(t) \cdot b_{\ell}\right|=\left|\varphi(t) \cdot b_{m}\right|\right\}
$$

are of zero measure for all $\ell \neq m$. For this to hold it is sufficient that all the pairs $\left(A, b_{\ell} \pm b_{m}\right)$ with $\ell \neq m$ satisfy the Kalman rank condition.

The corresponding controls are then of the form

$$
u_{j}(t)=\hat{\varphi}(t) \cdot b_{j} 1_{S_{j}}(t), \quad t \in(0, T), j=1, \ldots, p,
$$

where

$$
S_{j}=\left\{t \in(0, T):\left|\hat{\varphi}(t) \cdot b_{j}\right|>\left|\hat{\varphi}(t) \cdot b_{m}\right|, \forall m \neq j\right\} .
$$

Summarizing, the following holds:

Theorem 2.3. Assume that the pairs $\left(A, b_{\ell} \pm b_{m}\right)$ satisfy the Kalman condition for all $\ell, m=1, \ldots, p, \ell \neq m$. Then system 2.34) is controllable with switching controls satisfying

$$
u_{\ell}(t) u_{m}(t)=0, \quad \text { a.e. } t \in(0, T), \forall \ell \neq m .
$$

Furthermore, the controls are of the form 2.37-2.38 where $\hat{\varphi}$ is the solution of the adjoint system 2.6 associated to the minimizer $\hat{\varphi}^{0}{ }_{\text {of }} J_{s}$ in 2.35 . 
Furthermore, the controls may also be built to be of bang-bang form while the switching condition (2.39) is kept by minimizing the functional

$$
\begin{aligned}
J_{s b}\left(\varphi^{0}\right)= & \frac{1}{2}\left(\int_{0}^{T} \max \left(\left|\varphi(t) \cdot b_{1}\right|, \ldots,\left|\varphi(t) \cdot b_{p}\right|\right) d t\right)^{2} \\
& -\left\langle x^{1}, \varphi^{0}\right\rangle+x^{0} \cdot \varphi(0) .
\end{aligned}
$$

Vector valued controllers. Similar problems arise in the case where the controllers are not scalar. Let us consider, for instance, the more general system

$$
\left\{\begin{array}{l}
x^{\prime}(t)=A x(t)+B_{1} u_{1}(t)+B_{2} u_{2}(t) \\
x(0)=x^{0}
\end{array}\right.
$$

where $B_{1}$ and $B_{2}$ are $N \times M_{1}$ and $N \times M_{2}$ matrices and the controllers $u_{1}$ and $u_{2}$ are vectors of $M_{1}$ and $M_{2}$ components, respectively.

So far we have considered the particular case in which $M_{1}=M_{2}=1$. In the present case the functional to be minimized is

$$
J_{S}\left(\varphi^{0}\right)=\frac{1}{2} \int_{0}^{T} \max \left(\left|B_{1}^{*} \varphi(t)\right|^{2},\left|B_{2}^{*} \varphi(t)\right|^{2}\right) d t-x^{1} \cdot \varphi^{0}+x^{0} \cdot \varphi(0),
$$

where $B_{1}^{*} \varphi\left(\right.$ resp. $\left.B_{2}^{*} \varphi\right)$ are $M_{1}$ (resp. $M_{2}$ ) dimensional vectors and $|\cdot|$ stands for their euclidean norm. This functional has a minimizer when it is coercive and this holds when the pair $(A, B)$ satisfies the Kalman rank condition with $B=\left(B_{1}, B_{2}\right)$.

The minimizer yields a control of switching form if the set of time instants $t$ for which $\left|B_{1}^{*} \varphi(t)\right|=\left|B_{2}^{*} \varphi(t)\right|$ can be guaranteed to be of null measure.

In the case where $M_{1}=M_{2}=1$ and $B_{1}=b_{1}, B_{2}=b_{2}$ we have seen that the sharp condition to guarantee that the minimizer of $J_{S}$ yields switching controls is that $\left(A, B_{1} \pm B_{2}\right)$ satisfy the Kalman rank condition.

In the more general case under consideration we can use the Fourier expansion of solutions

$$
\varphi(t)=\sum_{\lambda} a_{\lambda} e^{\lambda t} w_{\lambda}
$$

$\lambda$ being the eigenvalues of $A$ and $w_{\lambda}$ the corresponding eigenvectors. Then

$$
\left|B_{j}^{*} \varphi(t)\right|^{2}=\sum_{k, \ell} a_{k} a_{\ell} e^{\left(\lambda_{k}+\lambda_{\ell}\right) t} \gamma_{k, \ell}^{j}
$$

where

$$
\gamma_{k, \ell}^{j}=\left\langle B_{j}^{*} w_{k}, B_{j}^{*} w_{\ell}\right\rangle, \quad j=1,2 .
$$

Arguing as above, in the case of time dependent switching strategies (see also Section 6.1) we see that a sufficient condition to guarantee that the controls obtained by minimizing $J_{S}$ be of switching form is that, for all $\Lambda$, if

$$
\sum_{\lambda_{k}+\lambda_{\ell}=\Lambda} a_{k} a_{\ell}\left[\gamma_{k, \ell}^{1}-\gamma_{k, \ell}^{2}\right]=0,
$$

then, necessarily, $\left\{a_{k}\right\} \equiv 0$. 
Assuming that

(H) the matrix $A$ is such that for each $\Lambda$, there is at most one pair of $(k, \ell)$ such that $\lambda_{k}+\lambda_{\ell}=\Lambda$,

it is easy to see that the condition above implies that

$$
a_{k}^{2}\left[\gamma_{k, k}^{1}-\gamma_{k, k}^{2}\right]=0, \quad \forall k .
$$

It is then sufficient to make sure that $\gamma_{k, k}^{1} \neq \gamma_{k, k}^{2}$ to ensure that all the coefficients $a_{k}$ vanish. In case this last condition fails for given control matrices $B_{1}$ and $B_{2}$ it is easy to see that it can be guaranteed to hold under suitable arbitrarily small perturbations of the matrices $B_{1}$ and $B_{2}$.

It would be of interest to investigate the class of matrices $A$ for which the spectral condition $(H)$ holds. We shall return to this matter below in the context of the multidimensional heat equation. As we shall see, according to the results in [15], the analogue of this assumption holds for the Dirichlet Laplacian generically with respect to the domain within the class of domains which are topological balls. It would also be of interest to see when system 2.43 implies that all the coefficients $a_{k}$ vanish in the absence of the condition $(H)$.

Of course, the spectrum of $A$ being simple is a necessary condition for $(H)$ to hold 2

\subsection{An example}

Let us now give an example of application. Consider the case where $A$ is the diagonal matrix

$$
A=\left(\begin{array}{ll}
1 & 0 \\
0 & 2
\end{array}\right)
$$

and the control vectors are

$$
b_{1}=\left(\begin{array}{l}
1 \\
0
\end{array}\right), \quad b_{2}=\left(\begin{array}{l}
0 \\
1
\end{array}\right) .
$$

It is easy to see that none of the pairs $\left(A, b_{1}\right)$ and $\left(A, b_{2}\right)$ fulfills the Kalman rank condition. The rank of the corresponding Kalman matrices is actually 1 . Thus, the system is not controllable with a single control, either $b_{1}$ or $b_{2}$. This is easy to predict because of the diagonal structure of the matrix $A$ that shows that the two components of the system are decoupled. This, together with the fact that the control vectors $b_{1}, b_{2}$ only excite one of the components, makes the controllability impossible.

However it is easy to see that the pairs $\left(A, b_{1} \pm b_{2}\right)$ do satisfy the Kalman rank condition. Consequently, the system is controllable under the action of the controls $b_{1} \pm b_{2}$.

Accordingly the hypotheses of 2.1 are satisfied and a switching control strategy may be built following the method of the previous subsection.

\footnotetext{
2 As pointed out to us by S. Ervedoza, when the eigenvalues are all real or purely imaginary this suffices to conclude under the assumption that $\gamma_{k, k}^{1} \neq \gamma_{k, k}^{2}$, for all $k$. For instance, if the eigenvalues are all real, we order them increasingly and then taking $\Lambda=\lambda_{1}$ we deduce that $a_{1}=0$. Taking next $\Lambda=\lambda_{1}+\lambda_{2}$ we conclude that $a_{2}=0$. Iterating this argument we can show that $a_{k}=0$ for all $k$.
} 

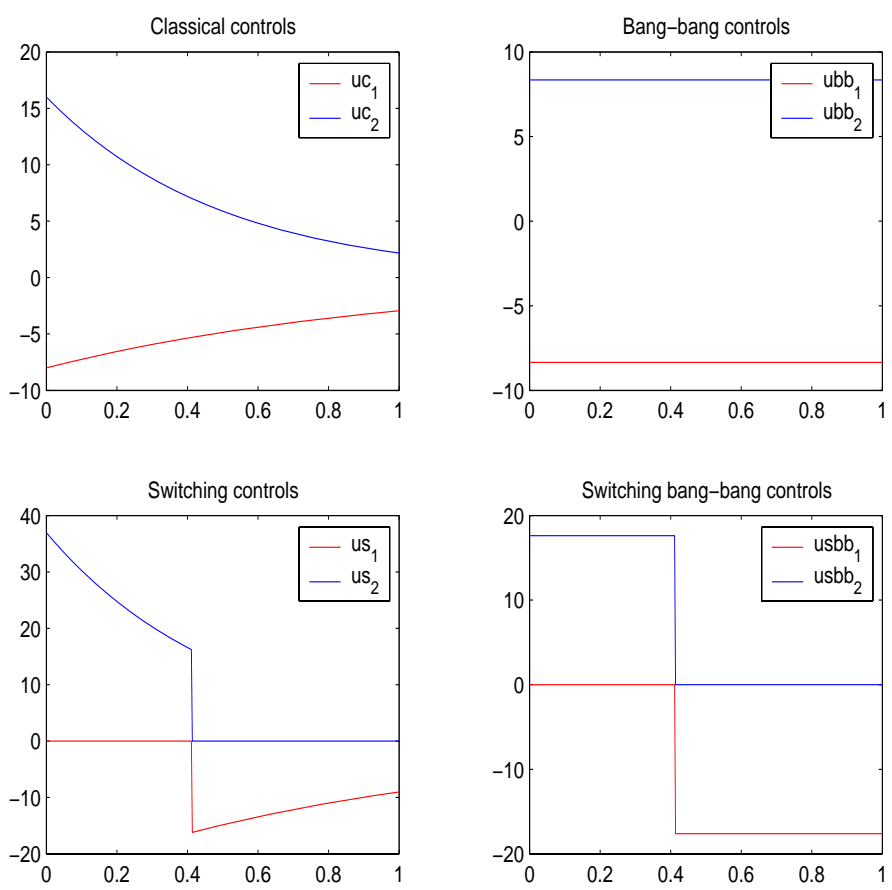

In the figure we draw the controls of this finite-dimensional system in four different situations. First (top left) we show the classical smooth controls obtained by minimizing the functional $J$ in 2.7). Then (top right) we present classical bang-bang controls. Then (bottom left) we draw the switching controls under discussion. Finally (bottom right) we plot the graph of switching bang-bang controls that we shall discuss below. All this is done in the case where the initial datum is $x_{0}=(2,-3)$ and the final datum $x_{T}=(-4,7)$.

\section{Relaxed switching controls}

In this section we discuss the output of the minimization of functionals of the form $J_{s}$ as in 2.26) when the condition guaranteeing that the pairs $\left(A, b_{1} \pm b_{2}\right)$ satisfy the Kalman rank condition fails. In this case the sets $I_{ \pm}$in 2.15, where $\tilde{\varphi}$ is a minimizer of $J_{s}$, cannot be guaranteed to be of null measure. In this section we discuss what the corresponding Euler-Lagrange equation and control result are.

The following holds:

Theorem 3.1. Assume that the pair $(A, B)$ with $B=\left(b_{1}, b_{2}\right)$ fulfills the Kalman rank condition. Then the functional $J_{s}$ in 2.10) is coercive. Moreover if $\tilde{\varphi}$ is a minimizer of $J_{S}$ then system (2.1) is controllable with controls $\left(u_{1}, u_{2}\right)$ such that

$$
\begin{cases}u_{1}(t)=\tilde{\varphi}(t) \cdot b_{1}, \quad u_{2}(t)=0 & \text { when }\left|\tilde{\varphi}(t) \cdot b_{1}\right|>\left|\tilde{\varphi}(t) \cdot b_{2}\right|, \\ u_{1}(t)=\frac{1}{2} \tilde{\varphi}(t) \cdot b_{1}, \quad u_{2}(t)=\frac{1}{2} \tilde{\varphi}(t) \cdot b_{2} & \text { when }\left|\tilde{\varphi}(t) \cdot b_{2}\right|=\left|\tilde{\varphi}(t) \cdot b_{1}\right|, \\ u_{1}(t)=0, \quad u_{2}(t)=\tilde{\varphi}(t) \cdot b_{2} & \text { when }\left|\tilde{\varphi}(t) \cdot b_{2}\right|>\left|\tilde{\varphi}(t) \cdot b_{1}\right| .\end{cases}
$$


In particular, both controls $u_{1}$ and $u_{2}$ are active on the set $\left|\tilde{\varphi}(t) \cdot b_{1}\right|=\left|\tilde{\varphi}(t) \cdot b_{2}\right|$, when it is of positive measure.

Observe that the controls we obtain in this case are not of switching form in a strict sense since the switching condition fails in the set $\left|\tilde{\varphi}(t) \cdot b_{1}\right|=\left|\tilde{\varphi}(t) \cdot b_{2}\right|$ where the amplitudes of the two positive quantities entering the quadratic term of the functional $J_{s}$ to be minimized coincide.

Proof. The proof is similar to the one of Theorem 2.1. We have, however, to pay special attention to the derivation of the Euler-Lagrange equations. The arguments developed in the proof of Theorem 2.1 apply within the set $\left|\tilde{\varphi}(t) \cdot b_{1}\right| \neq\left|\tilde{\varphi}(t) \cdot b_{2}\right|$ and yield the controls in 3.1). But we have to analyze the behavior of the functional within the set $\left|\tilde{\varphi}(t) \cdot b_{1}\right|=\left|\tilde{\varphi}(t) \cdot b_{2}\right|$. To do this we introduce some notation. Let $\tilde{\varphi}$ be a minimizer and set

$$
\left\{\begin{array}{l}
S_{1}=\left\{t \in(0, T):\left|\tilde{\varphi}(t) \cdot b_{1}\right|>\left|\tilde{\varphi}(t) \cdot b_{2}\right|\right\} \\
S_{-}=\left\{t \in(0, T): \tilde{\varphi}(t) \cdot\left(b_{1}-b_{2}\right)=0\right\} \\
S_{+}=\left\{t \in(0, T): \tilde{\varphi}(t) \cdot\left(b_{1}+b_{2}\right)=0\right\} \\
S_{2}=\left\{t \in(0, T):\left|\tilde{\varphi}(t) \cdot b_{1}\right|<\left|\tilde{\varphi}(t) \cdot b_{2}\right|\right\}
\end{array}\right.
$$

It is easy to see that $\tilde{\varphi}$, being a minimizer of $J_{S}$, is also a minimizer of the modified functional

$$
\begin{aligned}
\tilde{J}_{s}\left(\varphi^{0}\right)= & \frac{1}{2} \int_{S_{1} \cup S_{2}} \max \left(\left|b_{1} \cdot \varphi(t)\right|^{2},\left|b_{2} \cdot \varphi(t)\right|^{2}\right) d t \\
& +\frac{1}{4} \int_{S_{-} \cup S_{+}}\left[\left|b_{1} \cdot \varphi(t)\right|^{2}+\left|b_{2} \cdot \varphi(t)\right|^{2}\right] d t-x^{1} \cdot \varphi^{0}+x^{0} \cdot \varphi(0)
\end{aligned}
$$

over the set of solutions $\varphi$ such that

$$
\mathcal{X}=\left\{\varphi: \varphi(t) \cdot\left(b_{1}-b_{2}\right)=0 \text { in } S_{-} ; \varphi(t) \cdot\left(b_{1}+b_{2}\right)=0 \text { in } S_{+}\right\} .
$$

In this case there exist Lagrange multipliers $\lambda_{-}$and $\lambda_{+}$such that the Euler-Lagrange equations read

$$
\begin{aligned}
& \int_{S_{1}} \tilde{\varphi}(t) \cdot b_{1} \psi(t) \cdot b_{1} d t+\int_{S_{2}} \tilde{\varphi}(t) \cdot b_{2} \psi(t) \cdot b_{2} d t \\
& \quad+\frac{1}{2} \int_{S_{-} \cup S_{+}}\left[\tilde{\varphi}(t) \cdot b_{1} \psi(t) \cdot b_{1}+\tilde{\varphi}(t) \cdot b_{2} \psi(t) \cdot b_{2}\right] d t-x^{1} \cdot \psi^{0}+x^{0} \cdot \psi(0) \\
& =\lambda_{-} \int_{S_{-}} \tilde{\varphi}(t) \cdot\left(b_{1}-b_{2}\right) \psi(t) \cdot\left(b_{1}-b_{2}\right) d t+\lambda_{+} \int_{S_{+}} \tilde{\varphi}(t) \cdot\left(b_{1}+b_{2}\right) \psi(t) \cdot\left(b_{1}+b_{2}\right) d t .
\end{aligned}
$$

This means that the control defined as

$$
\begin{gathered}
u_{1}(t)=\tilde{\varphi}(t) \cdot b_{1} \quad \text { in } S_{1}, \\
u_{1}(t)=\left(1 / 2-\lambda_{-}\right) \tilde{\varphi}(t) \cdot b_{1}+\lambda_{-} \tilde{\varphi}(t) \cdot b_{2}, u_{2}(t)=\left(1 / 2-\lambda_{-}\right) \tilde{\varphi}(t) \cdot b_{2}+\lambda_{-} \tilde{\varphi}(t) \cdot b_{1} \quad \text { in } S_{-}, \\
u_{1}(t)=\left(1 / 2-\lambda_{+}\right) \tilde{\varphi}(t) \cdot b_{1}-\lambda_{+} \tilde{\varphi}(t) \cdot b_{2}, u_{2}(t)=\left(1 / 2-\lambda_{+}\right) \tilde{\varphi}(t) \cdot b_{2}-\lambda_{+} \tilde{\varphi}(t) \cdot b_{1} \quad \text { in } S_{+}, \\
u_{2}(t)=\tilde{\varphi}(t) \cdot b_{2} \quad \text { in } S_{2},
\end{gathered}
$$

fulfills the final requirement 2.2 . 
In view of the definition of the sets $S_{-}$and $S_{+}$and, more precisely, taking into account that $\tilde{\varphi}(t) \cdot b_{1}=\tilde{\varphi}(t) \cdot b_{2}$ in $S_{-}$and $\tilde{\varphi}(t) \cdot b_{1}=-\tilde{\varphi}(t) \cdot b_{2}$ in $S_{+}$we see that, on the set $S_{-} \cup S_{+}$, the controls take the form

$$
u_{1}(t)=\frac{1}{2} \tilde{\varphi}(t) \cdot b_{1}, \quad u_{2}(t)=\frac{1}{2} \tilde{\varphi}(t) \cdot b_{2}
$$

This completes the proof of the theorem.

\section{Optimality conditions}

We have built switching controllers by minimizing functionals $J_{s}$ of the form 2.26 or their variants. In this section we show that the controls obtained this way, in addition to being of switching form, are also optimal in some sense.

In this respect it is worth mentioning that by now it is well known that the controls obtained when minimizing functionals of the form (2.7) yield controls of minimal $L^{2}\left(0, T ; \mathbb{R}^{2}\right)$-norm, while those obtained when replacing 2.7 by

$$
J_{b b}\left(\varphi^{0}\right)=\frac{1}{2}\left[\int_{0}^{T}\left(\left|\varphi(t) \cdot b_{1}\right|+\left|\varphi(t) \cdot b_{2}\right|\right) d t\right]^{2}-x^{1} \cdot \varphi^{0}+x^{0} \cdot \varphi(0)
$$

yield bang-bang controls which turn out to be of minimal $L^{\infty}\left(0, T ; \mathbb{R}^{2}\right)$-norm. The latter can be proved using the Fenchel-Rocafellar duality principle as in [3] or using directly the characterization of the controls as minimizers of $J_{b b}$ (see [13]).

In the present setting the following holds:

Theorem 4.1. Let the assumptions of Theorem 2.1 be satisfied. Then the controls $\left(\tilde{u}_{1}, \tilde{u}_{2}\right)$ obtained by minimizing the functional $J_{s}$ in 2.26 , in addition to satisfying the switching condition 2.4), are of minimal norm in $L^{2}\left(0, T ; \mathbb{R}^{2}\right)$, the space $\mathbb{R}^{2}$ being endowed with the $\ell^{1}$-norm. More precisely,

$$
\int_{0}^{T}\left(\left|\tilde{u}_{1}\right|+\left|\tilde{u}_{2}\right|\right)^{2} d t \leq \int_{0}^{T}\left(\left|u_{1}\right|+\left|u_{2}\right|\right)^{2} d t
$$

for all other admissible control pairs $\left(u_{1}, u_{2}\right)$ satisfying the final requirement 2.2 .

Proof. We first observe that, in view of the fact that the controls $\left(\tilde{u}_{1}, \tilde{u}_{2}\right)$ are of switching form, it follows that

$$
\left|\tilde{u}_{1}(t)\right|+\left|\tilde{u}_{2}(t)\right|=\max \left(\left|\tilde{u}_{1}(t)\right|,\left|\tilde{u}_{2}(t)\right|\right), \quad \forall t \in(0, T) .
$$

Let $\left(u_{1}, u_{2}\right)$ be any other pair of switching controls. Multiplying the state equation 2.1 by $\tilde{\varphi}$, the solution of the adjoint system associated to the minimizer of $J_{s}$, both for the 
controls $\left(u_{1}, u_{2}\right)$ and $\left(\tilde{u}_{1}, \tilde{u}_{2}\right)$, and using the fact that in both cases the final requirement 2.2 is satisfied, we deduce that

$$
\begin{aligned}
\left\langle x^{1}, \tilde{\varphi}^{0}\right\rangle-\left\langle x^{0}, \tilde{\varphi}(0)\right\rangle & =\int_{0}^{T}\left(u_{1}(t) b_{1} \cdot \tilde{\varphi}(t)+u_{2}(t) b_{2} \cdot \tilde{\varphi}(t)\right) d t, \\
\left\langle x^{1}, \tilde{\varphi}^{0}\right\rangle-\left\langle x^{0}, \tilde{\varphi}(0)\right\rangle & =\int_{0}^{T}\left(\tilde{u}_{1}(t) b_{1} \cdot \tilde{\varphi}(t)+\tilde{u}_{2}(t) b_{2} \cdot \tilde{\varphi}(t)\right) d t .
\end{aligned}
$$

In view of the very nature of the switching controls $\left(\tilde{u}_{1}, \tilde{u}_{2}\right)$ we deduce that

$$
\begin{aligned}
\int_{0}^{T}\left(\tilde{u}_{1}(t) b_{1} \cdot \tilde{\varphi}(t)+\tilde{u}_{2}(t) b_{2} \cdot \tilde{\varphi}(t)\right) d t & =\int_{0}^{T} \max \left(b_{1} \cdot \tilde{\varphi}(t), b_{2} \cdot \tilde{\varphi}(t)\right)^{2} d t \\
& =\int_{0}^{T}\left(\left|\tilde{u}_{1}\right|+\left|\tilde{u}_{2}\right|\right)^{2} d t
\end{aligned}
$$

Combining these identities we deduce that

$$
\begin{aligned}
\int_{0}^{T}\left(\left|\tilde{u}_{1}\right|+\left|\tilde{u}_{2}\right|\right)^{2} d t & =\int_{0}^{T}\left(u_{1}(t) b_{1} \cdot \tilde{\varphi}(t)+u_{2}(t) b_{2} \cdot \tilde{\varphi}(t)\right) d t \\
& \leq \int_{0}^{T}\left(\left|u_{1}(t)\right|+\left|u_{2}(t)\right|\right) \max \left(\left|b_{1} \cdot \tilde{\varphi}(t)\right|,\left|b_{2} \cdot \tilde{\varphi}(t)\right|\right) d t \\
& =\int_{0}^{T}\left(\left|u_{1}(t)\right|+\left|u_{2}(t)\right|\right) \max \left(\left|\tilde{u}_{1}(t)\right|,\left|\tilde{u}_{2}(t)\right|\right) d t \\
& =\int_{0}^{T}\left(\left|u_{1}(t)\right|+\left|u_{2}(t)\right|\right)\left(\left|\tilde{u}_{1}(t)\right|+\left|\tilde{u}_{2}(t)\right|\right) d t \\
& \leq\left[\int_{0}^{T}\left(\left|u_{1}(t)\right|+\left|u_{2}(t)\right|\right)^{2} d t\right]^{1 / 2}\left[\int_{0}^{T}\left(\left|\tilde{u}_{1}(t)\right|+\left|\tilde{u}_{2}(t)\right|\right)^{2} d t\right]^{1 / 2}
\end{aligned}
$$

which implies that

$$
\int_{0}^{T}\left(\left|\tilde{u}_{1}\right|+\left|\tilde{u}_{2}\right|\right)^{2} d t \leq \int_{0}^{T}\left(\left|u_{1}(t)\right|+\left|u_{2}(t)\right|\right)^{2} d t
$$

as desired.

Remark 4.1. - Similar arguments allow showing that the bang-bang switching controllers we have built by minimizing the functional $J_{s b}$ in 2.31 are minimal in the sense of the $L^{\infty}\left(0, T ; \ell^{1}\right)$-norm.

- Also the same ideas and results apply when the number of controllers is greater than two.

- The same optimality condition holds for the relaxed switching controls obtained in Theorem 3.1 under the weaker condition guaranteeing that the pair $(A, B)$ with $B=$ $\left(b_{1}, b_{2}\right)$ fulfills the Kalman rank condition. 
Once more we have

$$
\begin{aligned}
\left\langle x^{1}, \tilde{\varphi}^{0}\right\rangle-\left\langle x^{0}, \tilde{\varphi}(0)\right\rangle & =\int_{0}^{T}\left(u_{1}(t) b_{1} \cdot \tilde{\varphi}(t)+u_{2}(t) b_{2} \cdot \tilde{\varphi}(t)\right) d t, \\
\left\langle x^{1}, \tilde{\varphi}^{0}\right\rangle-\left\langle x^{0}, \tilde{\varphi}(0)\right\rangle & =\int_{0}^{T}\left(\tilde{u}_{1}(t) b_{1} \cdot \tilde{\varphi}(t)+\tilde{u}_{2}(t) b_{2} \cdot \tilde{\varphi}(t)\right) d t,
\end{aligned}
$$

and, in view of the very nature of the minimizing controls $\left(\tilde{u}_{1}, \tilde{u}_{2}\right)$,

$$
\int_{0}^{T}\left(\tilde{u}_{1}(t) b_{1} \cdot \tilde{\varphi}(t)+\tilde{u}_{2}(t) b_{2} \cdot \tilde{\varphi}(t)\right) d t=\int_{0}^{T}\left(\left|\tilde{u}_{1}\right|+\left|\tilde{u}_{2}\right|\right)^{2} d t .
$$

Combining these identities we deduce that

$$
\begin{aligned}
\int_{0}^{T}\left(\left|\tilde{u}_{1}\right|+\left|\tilde{u}_{2}\right|\right)^{2} d t & =\int_{0}^{T}\left(u_{1}(t) b_{1} \cdot \tilde{\varphi}(t)+u_{2}(t) b_{2} \cdot \tilde{\varphi}(t)\right) d t \\
& \leq \int_{0}^{T}\left(\left|u_{1}(t)\right|+\left|u_{2}(t)\right|\right) \max \left(\left|b_{1} \cdot \tilde{\varphi}(t)\right|,\left|b_{2} \cdot \tilde{\varphi}(t)\right|\right) d t \\
& =\int_{0}^{T}\left(\left|u_{1}(t)\right|+\left|u_{2}(t)\right|\right)\left(\left|\tilde{u}_{1}(t)\right|+\left|\tilde{u}_{2}(t)\right|\right) d t \\
& \leq\left[\int_{0}^{T}\left(\left|u_{1}(t)\right|+\left|u_{2}(t)\right|\right)^{2} d t\right]^{1 / 2}\left[\int_{0}^{T}\left(\left|\tilde{u}_{1}(t)\right|+\left|\tilde{u}_{2}(t)\right|\right)^{2} d t\right]^{1 / 2},
\end{aligned}
$$

which implies the optimality of the controls $\left(\tilde{u}_{1}, \tilde{u}_{2}\right)$.

\section{The 1-d heat equation}

The 1-d heat equation, due to its particularly simple geometry and the time analyticity of its solutions, is the model more closely related to the finite-dimensional systems we have considered in the previous section. Certainly the most natural problem to consider in this case is that of switching between two boundary controls. But, as we shall see, even though switching controls trivially exist, we have to carefully adapt our strategy to develop a systematic variational way of computing them, due to the space symmetry of the problem. To break this symmetry, it is also natural to consider the problem of two pointwise actuators, placed at different points of the domain where the equation evolves. As we shall see, our methods apply in that case under suitable irrationality conditions on the points where the controls are placed.

\subsection{Boundary controls}

Consider the heat equation in the space interval $(0,1)$ with two controls located at the extremes $x=0,1$ :

$$
\begin{cases}y_{t}-y_{x x}=0, & 0<x<1,0<t<T \\ y(0, t)=u_{0}(t), \quad y(1, t)=u_{1}(t), & 0<t<T \\ y(x, 0)=y^{0}(x), & 0<x<1\end{cases}
$$


We consider the problem of null controllability. More precisely, given an initial datum $y^{0} \in L^{2}(0,1)$ we look for controls $u_{0}, u_{1} \in L^{2}(0, T)$ such that the solution of 5.1. satisfies

$$
y(x, T) \equiv 0 .
$$

It is by now well known that system [5.1] is null controllable (we refer to [19] for a recent survey on this topic both in one and several space dimensions). In fact, the system is controllable by means of one single control: either $u_{0}$ located at $x=0$ or $u_{1}$ located at $x=1$.

Here we are interested in switching controllers such that

$$
u_{0}(t) u_{1}(t)=0, \quad \text { a.e. } t \in(0, T) .
$$

Obviously, switching controllers exist since any of the controls $u_{0}$ and $u_{1}$ by itself suffices to control the system and therefore one could take, for instance, $u_{1} \equiv 0$, which clearly fulfills [5.3. In the finite-dimensional context of the previous section, this would correspond to the situation in which both the pairs $\left(A, b_{1}\right)$ and $\left(A, b_{2}\right)$ fulfill the Kalman rank condition.

We now analyze whether in the present context the methodology that we have described in the previous section can be adapted to give a systematic way of building those controls of switching form.

To do this we consider the adjoint system

$$
\begin{cases}\varphi_{t}+\varphi_{x x}=0, & 0<x<1,0<t<T, \\ \varphi(0, t)=\varphi(1, t)=0, & 0<t<T, \\ \varphi(x, T)=\varphi^{0}(x), & 0<x<1 .\end{cases}
$$

The following observability inequality for the solutions of (5.4) is well known:

$$
\|\varphi(x, 0)\|_{L^{2}(0,1)}^{2} \leq C \int_{0}^{T}\left[\left|\varphi_{x}(0, t)\right|^{2}+\left|\varphi_{x}(1, t)\right|^{2}\right] d t .
$$

Actually, the same is true if the right hand side of 5.5 only involves the $L^{2}(0, T)$-norm of $\varphi_{x}(0, t)$ or $\varphi_{x}(1, t)$.

It is also well known that the null control of 5.1) may be computed by minimizing the quadratic functional

$$
J\left(\varphi^{0}\right)=\frac{1}{2} \int_{0}^{T}\left[\left|\varphi_{x}(0, t)\right|^{2}+\left|\varphi_{x}(1, t)\right|^{2}\right] d t+\int_{0}^{1} y^{0}(x) \varphi(x, 0) d x
$$

over the class $\mathcal{H}$ of initial data given by

$$
\mathcal{H}=\left\{\varphi^{0}: \int_{0}^{T}\left[\left|\varphi_{x}(0, t)\right|^{2}+\left|\varphi_{x}(1, t)\right|^{2}\right] d t<\infty\right\}
$$

which, endowed with the canonical norm

$$
\left\|\varphi^{0}\right\|_{\mathcal{H}}=\left[\int_{0}^{T}\left(\left|\varphi_{x}(0, t)\right|^{2}+\left|\varphi_{x}(1, t)\right|^{2}\right) d t\right]^{1 / 2}
$$

constitutes a Hilbert space. 
The controls obtained this way take the form

$$
u_{0}(t)=-\hat{\varphi}_{x}(0, t), \quad u_{1}(t)=\hat{\varphi}_{x}(1, t), \quad t \in(0, T),
$$

where $\hat{\varphi}$ is the solution of (5.4) associated to the minimizer $\hat{\varphi}^{0} \in \mathcal{H}$ of $J$.

Obviously these controls, generically, do not fulfill the switching conditions 5.3 .

In view of the results of the previous section, when looking for switching controls, it is natural to consider the functional

$$
J_{S}\left(\varphi^{0}\right)=\frac{1}{2} \int_{0}^{T} \max \left[\left|\varphi_{x}(0, t)\right|^{2},\left|\varphi_{x}(1, t)\right|^{2}\right] d t+\int_{0}^{1} y^{0}(x) \varphi(x, 0) d x .
$$

This functional $J_{S}: \mathcal{H} \rightarrow \mathbb{R}$ is well defined, continuous and convex. It is also coercive in view of the observability inequality 5.5 . Consequently, its minimizer exists: $\hat{\varphi}^{0} \in$ $L^{2}(0,1)$.

It is natural to analyze whether the controls are of switching form

$$
u_{0}(t)=\hat{\varphi}_{x}(0, t) 1_{S_{0}}(t), \quad u_{1}(t)=\hat{\varphi}_{x}(1, t) 1_{S_{1}}(t),
$$

$\hat{\varphi}$ being the solution of 5.4 associated to the initial datum $\hat{\varphi}^{0}$, and $S_{0}$ and $S_{1}$ being, respectively, the sets where $\left|\hat{\varphi}_{x}(0, t)\right|>\left|\hat{\varphi}_{x}(1, t)\right|$ and $\left|\hat{\varphi}_{x}(0, t)\right|<\left|\hat{\varphi}_{x}(1, t)\right|$.

For this to be true and to guarantee that the controls obtained this way are of switching form, one has to check that the set

$$
I=\left\{t \in(0, T):\left|\hat{\varphi}_{x}(0, t)\right|=\left|\hat{\varphi}_{x}(1, t)\right|\right\}
$$

is of zero measure, which is equivalent to the following two sets being of null measure:

$$
I_{ \pm}=\left\{t \in(0, T): \hat{\varphi}_{x}(0, t)= \pm \hat{\varphi}_{x}(1, t)\right\}
$$

But this may not be guaranteed to hold. Actually, the following unique continuation properties are clearly false:

$$
\left[\varphi_{x}(0, t)= \pm \varphi_{x}(1, t) \text { for a.e. } t \in I_{ \pm} \text {, and }\left|I_{ \pm}\right|>0\right] \Rightarrow \varphi \equiv 0 .
$$

Indeed, every even (with respect to the center $x=1 / 2$ ) solution is a counterexample with the - sign, while odd solutions show that the result fails for the + sign too.

In the finite-dimensional setting of the previous section this would correspond to the failure of the property that the pairs $\left(A, b_{1}-b_{2}\right)$ and $\left(A, b_{1}+b_{2}\right)$ satisfy the Kalman rank condition. In our setting this corresponds to the fact that system (5.1) is not null controllable with controls $\left(u_{0}, u_{1}\right)$ satisfying either $u_{0}=u_{1}$ or $u_{0}=-u_{1}$, because of the symmetry properties of the system.

In this case applying the arguments of Section 3.1 we can show that the controls obtained by minimizing the functional $J$ have a generalized switching form, namely

$$
\begin{cases}u_{0}(t)=-\hat{\varphi}_{x}(0, t), \quad u_{1}(t)=0 & \text { when }\left|\hat{\varphi}_{x}(0, t)\right|>\left|\hat{\varphi}_{x}(1, t)\right|, \\ u_{0}(t)=-\frac{1}{2} \hat{\varphi}_{x}(0, t), \quad u_{1}(t)=\frac{1}{2} \hat{\varphi}_{x}(1, t) & \text { when }\left|\hat{\varphi}_{x}(0, t)\right|=\left|\hat{\varphi}_{x}(1, t)\right|, \\ u_{0}(t)=0, \quad u_{1}(t)=\hat{\varphi}_{x}(1, t) & \text { when }\left|\hat{\varphi}_{x}(1, t)\right|>\left|\hat{\varphi}_{x}(0, t)\right|\end{cases}
$$


Arguing as in the previous section, we can consider a more general functional of the form

$$
J_{s}^{\alpha}\left(\varphi^{0}\right)=\frac{1}{2} \int_{0}^{T} \max \left[\left|\varphi_{x}(0, t)\right|^{2},\left|\alpha \varphi_{x}(1, t)\right|^{2}\right] d t+\int_{0}^{1} y^{0}(x) \varphi(x, 0) d x .
$$

By the same arguments, a minimizer exists. To guarantee that it is of switching form we have to make sure that the unique solution satisfying

$$
\varphi_{x}(0, t)= \pm \alpha \varphi_{x}(1, t)
$$

in a set of positive measure is the trivial one.

Let us analyze this issue. For this, it is convenient to use the Fourier representation of solutions of (5.4).

If $\varphi^{0} \in L^{2}(0,1)$ has the Fourier expansion

$$
\varphi^{0}(x)=\sum_{k \geq 1} \beta_{k} w_{k}(x)
$$

with

$$
w_{k}(x)=\sqrt{2} \sin (k \pi x),
$$

then the solution $\varphi$ of 5.4 is of the form

$$
\varphi(x, t)=\sum_{k \geq 1} \beta_{k} e^{k^{2}(t-T)} w_{k}(x)
$$

Then

$$
\varphi_{x}(0, t) \pm \varphi_{x}(1, t)=\sum_{k=1}^{\infty} \beta_{k} e^{k^{2}(t-T)}\left(w_{k, x}(0) \pm \alpha w_{k, x}(1)\right) .
$$

The functions $\varphi_{x}(0, t) \pm \alpha \varphi_{x}(1, t)$ are time analytic for $t \leq T$. Consequently, if they vanish for a set of time instants of positive measure, then they vanish for all $t \leq T$. It is then easy to see, multiplying in 5.17 by the real exponentials $e^{-\ell^{2}(t-T)}$ successively, starting from $\ell=1$, and taking limits as $t \rightarrow-\infty$, that

$$
\beta_{k}\left(w_{k, x}(0) \pm \alpha w_{k, x}(1)\right)=0, \quad \forall k \geq 1 .
$$

To conclude that $\beta_{k}=0$ for all $k \geq 1$ it is sufficient to show that

$$
w_{k, x}(0) \pm \alpha w_{k, x}(1)=\sqrt{2} k \pi(1 \pm \alpha \cos (k \pi)) \neq 0, \quad \forall k \geq 1,
$$

which is obviously true if $|\alpha| \neq 1$.

We have proved the following result:

Theorem 5.1. By minimizing $J_{s}^{\alpha}$ with $|\alpha| \neq 1$ we obtain switching controls.

A similar argument can be developed when the weight $\alpha=\alpha(t)$ in the definition of $J_{s}^{\alpha}$ depends on time and is analytic for $t \leq T$. In that case it is sufficient that, as $t \rightarrow-\infty$, the set of accumulation points of $\{\alpha(t)\}$ contains a value $\alpha$ such that $|\alpha| \neq 1$, since the spectrum is obviously simple. But it is not necessary to add such complexity to the functional since, as we have seen, taking a constant $\alpha$ with $|\alpha| \neq 1$ suffices. 


\subsection{Pointwise controls}

It is also natural to consider the case in which two pointwise controllers act at two different points $a$ and $b$ of the space interval $(0,1)$ where the equation is satisfied.

The corresponding control system is

$$
\begin{cases}y_{t}-y_{x x}=u_{a}(t) \delta_{a}+u_{b}(t) \delta_{b}, & 0<x<1,0<t<T, \\ y(0, t)=y(1, t)=0, & 0<t<T, \\ y(x, 0)=y^{0}(x), & 0<x<1 .\end{cases}
$$

The quadratic functional associated to the adjoint system for this control problem is as follows:

$$
J_{S}\left(\varphi^{0}\right)=\frac{1}{2} \int_{0}^{T} \max \left(|\varphi(a, t)|^{2},|\varphi(b, t)|^{2}\right) d t-\int_{0}^{1} y^{0}(x) \varphi(x, 0) d x .
$$

There are two main issues to address:

- The coercivity of the functional $J_{s}$ in an appropriate space. This is closely related to whether the quantity

$$
\left\|\varphi^{0}\right\|_{\mathcal{H}}=\left[\int_{0}^{T}\left[|\varphi(a, t)|^{2}+|\varphi(b, t)|^{2}\right] d t\right]^{1 / 2}
$$

defines a norm, and what additional information we can get from it.

- Whether the condition

$$
\varphi(a, t)+\varphi(b, t)=0 \quad(\text { or } \varphi(a, t)-\varphi(b, t)=0)
$$

over a set of positive measure guarantees that the corresponding solution $\varphi$ is trivial.

The second issue is essential to conclude that the controls that one may possibly obtain by minimizing the functional $J_{s}$ in (5.19) satisfy the switching condition. Let us first analyze that issue.

Using again the Fourier representation of solutions of 5.16 we have

$$
\varphi(a, t) \pm \varphi(b, t)=\sum_{k=1}^{\infty} \beta_{k} e^{k^{2}(t-T)}\left(w_{k}(a) \pm w_{k}(b)\right) .
$$

The functions $\varphi(a, t) \pm \varphi(b, t)$ are time analytic for $t \leq T$. Consequently, if they vanish for a set of time instants of positive measure, then they vanish for all $t \leq T$. It is then easy to see, multiplying in 5.22 by the real exponentials $e^{-k^{2}(t-T)}$ and taking limits as $t \rightarrow-\infty$, that

$$
\beta_{k}\left(w_{k}(a) \pm w_{k}(b)\right)=0, \quad \forall k \geq 1 .
$$

To conclude that $\beta_{k}=0$ for all $k \geq 1$ it is sufficient to show that

$$
w_{k}(a) \pm w_{k}(b)=\sin (k \pi a) \pm \sin (k \pi b) \neq 0, \quad \forall k \geq 1 .
$$


This holds if and only if

$$
k \pi a \pm k \pi b \neq m \pi, \quad \forall k \geq 1, m \in \mathbb{Z},
$$

i.e.

$$
a \pm b \neq m / k, \quad \forall k \geq 1, m \in \mathbb{Z} .
$$

In other words, a necessary and sufficient condition for the switching property to hold is that $a \pm b$ be irrational. This is, in the present setting, the analogue to the property that the pairs $\left(A, b_{1} \pm b_{2}\right)$ satisfy the Kalman rank condition in the finite-dimensional case.

As in Section 3.1. when condition 5.23 fails the controls obtained are of relaxed switching form.

Note that condition 5.23 implies that $a$ and/or $b$ are irrational and then system 5.18 is controllable with controls located only at $x=a$ or $x=b$. Indeed, the heat equation

$$
\begin{cases}y_{t}-y_{x x}=u_{a}(t) \delta_{a}, & 0<x<1,0<t<T, \\ y(0, t)=y(1, t)=0, & 0<t<T, \\ y(x, 0)=y^{0}(x), & 0<x<1,\end{cases}
$$

is controllable if and only if $a$ is irrational. In fact, when $a$ is rational the system fails to be approximately controllable 3 since the following unique continuation property fails for the solutions of the adjoint system:

$$
[\varphi(a, t)=0,0<t<T] \Rightarrow \varphi \equiv 0 .
$$

Let us finally analyze the class of initial data for system (5.18) for which switching controllers exist. By duality it is sufficient to analyze the positivity of the norm $\|\cdot\|_{\mathcal{H}}$ in 5.20 .

We have

$$
\begin{aligned}
\left\|\varphi^{0}\right\|_{\mathcal{H}}^{2} & =\int_{0}^{T}\left[|\varphi(a, t)|^{2}+|\varphi(b, t)|^{2}\right] d t \\
& =\int_{0}^{T}\left[\left|\sum_{k \geq 1} \beta_{k} e^{k^{2}(t-T)} w_{k}(a)\right|^{2}+\left|\sum_{k \geq 1} \beta_{k} e^{k^{2}(t-T)} w_{k}(b)\right|^{2}\right] d t .
\end{aligned}
$$

We can now use well known estimates on families of real exponentials that in this case guarantee that (see [12])

$$
\int_{0}^{T}\left|\sum_{k \geq 1} \beta_{k} e^{k^{2}(t-T)}\right|^{2} d t \geq c_{1} \sum_{k \geq 1} e^{-c_{2} k^{2} T} \alpha_{k}^{2}
$$

for suitable positive constants $c_{1}, c_{2}>0$ independent of $\left\{\beta_{k}\right\}_{k \geq 1}$.

3 The property of approximate controllability consists in driving the solution to an $\epsilon$ neighborhood of an arbitrary target $y^{1} \in L^{2}(0,1)$, with $\epsilon>0$ arbitrarily small. Null controllability is a stronger property which, roughly, ensures that the controls remain bounded in the corresponding norm as $\epsilon \rightarrow 0$ for the target $y^{1} \equiv 0$. 
We then get the weighted observability inequality

$$
\left\|\varphi^{0}\right\|_{\mathcal{H}}^{2} \geq c_{1} \sum_{k \geq 1} e^{-c_{2} k^{2} T}\left[\left|w_{k}(a)\right|^{2}+\left|w_{k}(b)\right|^{2}\right] \alpha_{k}^{2}
$$

The space of null controllable initial data is the dual one $\mathcal{H}^{\prime}$.

Summarizing, the following holds:

Theorem 5.2. Assume that $a$ and $b$ in the interval $(0,1)$ are such that the irrationality conditions 5.23 hold. Let the initial datum $y^{0}$ be in $\mathcal{H}^{\prime}$. More precisely, let $y^{0}$ be of the form

$$
y^{0}(x)=\sum_{k \geq 1} y_{k}^{0} w_{k}(x)
$$

with

$$
\sum_{k \geq 1} \frac{e^{c_{2} k^{2} T}}{\left|w_{k}(a)\right|^{2}+\left|w_{k}(b)\right|^{2}}\left|y_{k}^{0}\right|^{2}<\infty .
$$

Then, for all $T>0$, there exist switching controls $u_{a}$ and $u_{b}$ such that

$$
u_{a}(t) u_{b}(t)=0, \quad \text { a.e. } t \in(0, T),
$$

and that the solution of 5.18 satisfies

$$
y(T) \equiv 0 .
$$

These controls can be obtained by minimizing the functional 5.19.

Remark 5.1. How positive the weights in 5.25) are as $k \rightarrow \infty$, which determines the nature of the space of controllable data, depends strongly on the Diophantine properties of the irrational numbers $a$ and $b$. This issue often arises in 1-d control problems. We refer for instance to [2] for a discussion of this issue in the context of the control of wave processes on 1-d networks.

\subsection{Lumped controls}

Similar results hold in the case of lumped controls, in which the pointwise Dirac controls of the previous section are replaced by controls distributed by means of given control functions. To be more precise, let $f_{0}=f_{0}(x)$ and $f_{1}=f_{1}(x)$ be two control profiles and consider the heat equation

$$
\begin{cases}y_{t}-y_{x x}=u_{0}(t) f_{0}(x)+u_{1}(t) f_{1}(x), & 0<x<1,0<t<T, \\ y(0, t)=y(1, t)=0, & 0<t<T, \\ y(x, 0)=y^{0}(x), & 0<x<1 .\end{cases}
$$

Assume that the controls $f_{0}$ and $f_{1}$ have Fourier series expansions of the form

$$
f_{0}(x)=\sum_{k \geq 1} f_{0, k} w_{k}(x), \quad f_{1}(x)=\sum_{k \geq 1} f_{1, k} w_{k}(x)
$$


Then the system 5.26 is null controllable with switching controls under the condition

$$
f_{0, k} \pm f_{1, k} \neq 0, \quad \forall k \geq 1
$$

which is the analogue of the condition 5.23 in the case of pointwise controllers.

As in Section 3.1, when condition (5.28) fails the controls obtained are of relaxed switching form.

The initial data that can be controlled this time have Fourier coefficients $\left\{y_{k}^{0}\right\}$ satisfying

$$
\sum_{k \geq 1} \frac{e^{c_{2} k^{2} T}}{\left|f_{0, k}\right|^{2}+\left|f_{1, k}\right|^{2}}\left|y_{k}^{0}\right|^{2}<\infty .
$$

The controls can be obtained by minimizing the functional

$$
\begin{aligned}
J_{S}\left(\varphi^{0}\right)= & \frac{1}{2} \int_{0}^{T} \max \left(\left|\int_{0}^{1} f_{0}(x) \varphi(x, t) d x\right|^{2},\left|\int_{0}^{1} f_{1}(x) \varphi(x, t) d x\right|^{2}\right) d t \\
& -\int_{0}^{1} y^{0}(x) \varphi(x, 0) d x .
\end{aligned}
$$

\section{The multi-dimensional heat equation}

The techniques we have developed in the previous section can also be applied in the multidimensional case. However, the non-degeneracy conditions that need to be imposed are this time less explicit because they depend on the spectrum of the underlying elliptic operator. We illustrate this fact in the most commonly considered cases: boundary and internal controls.

\subsection{Internal controls}

Let $\Omega$ be a bounded smooth subset of $\mathbb{R}^{d}$ with $d \geq 1$ and $\omega_{1}, \omega_{2}$ be two subsets of $\Omega$. Consider the controlled heat equation

$$
\begin{cases}y_{t}-\Delta y=u_{1}(x, t) 1_{\omega_{1}}+u_{2}(x, t) 1_{\omega_{2}} & \text { in } Q=\Omega \times(0, T) \\ y=0 & \text { on } \Sigma=\partial Q \\ y(x, 0)=y^{0}(x) & \text { in } \Omega\end{cases}
$$

We assume that $y^{0} \in L^{2}(\Omega)$ and $u_{1}, u_{2} \in L^{2}(\Omega \times(0, T))$ so that 6.1 admits a unique solution

$$
y \in C\left([0, T] ; L^{2}(\Omega)\right) \cap L^{2}\left(0, T ; H_{0}^{1}(\Omega)\right) .
$$

We consider the problem of null controllability with switching controls. Thus, we look for $u_{1}$ and $u_{2}$ such that the solution of (6.1) satisfies

$$
y(T) \equiv 0,
$$


and, furthermore,

$$
\left\|u_{1}(t)\right\|_{L^{2}\left(\omega_{1}\right)}\left\|u_{2}(t)\right\|_{L^{2}\left(\omega_{2}\right)}=0, \quad \text { a.e. } t \in(0, T) .
$$

Condition (6.3) guarantees that, at each time instant $t$, only one control is activated.

To analyze the existence of such controls, and to build them whenever they exist, we consider the functional

$$
J_{S}\left(\varphi^{0}\right)=\frac{1}{2} \int_{0}^{T} \max \left(\int_{\omega_{1}}|\varphi(x, t)|^{2} d x, \int_{\omega_{2}}|\varphi(x, t)|^{2} d x\right) d t-\int_{0}^{1} y^{0}(x) \varphi(x, 0) d x,
$$

where $\varphi$ satisfies the adjoint system

$$
\begin{cases}\varphi_{t}+\Delta \varphi=0 & \text { in } Q, \\ \varphi=0 & \text { on } \Sigma, \\ \varphi(x, T)=\varphi^{0}(x) & \text { in } \Omega .\end{cases}
$$

Arguing as in the previous sections and using the existing observability inequalities, it is easy to see that the functional $J_{s}: L^{2}(\Omega) \rightarrow \mathbb{R}$, which is continuous and convex, is coercive too. The relevant observability inequality is

$$
\|\varphi(0)\|_{L^{2}(\Omega)}^{2} \leq C \int_{0}^{T} \int_{\omega_{1} \cup \omega_{2}} \varphi^{2} d x d t .
$$

We claim that, minimizing this functional, one obtains the switching controls

$$
u_{1}(x, t)=\hat{\varphi}_{1}(x, t) 1_{S_{1}}, \quad u_{2}(x, t)=\hat{\varphi}_{2}(x, t) 1_{S_{2}}
$$

where

$$
\begin{aligned}
& S_{1}=\left\{t \in(0, T):\|\hat{\varphi}(t)\|_{L^{2}\left(\omega_{1}\right)}>\|\hat{\varphi}(t)\|_{L^{2}\left(\omega_{2}\right)}\right\}, \\
& S_{2}=\left\{t \in(0, T):\|\hat{\varphi}(t)\|_{L^{2}\left(\omega_{2}\right)}>\|\hat{\varphi}(t)\|_{L^{2}\left(\omega_{1}\right)}\right\} .
\end{aligned}
$$

But for this to be true we have to show that the set

$$
I=\left\{t \in(0, T):\|\varphi(t)\|_{L^{2}\left(\omega_{1}\right)}=\|\varphi(t)\|_{L^{2}\left(\omega_{2}\right)}\right\}
$$

is of null measure whenever $\varphi$ is non-trivial.

Let us now discuss this condition. Using the time analyticity of the functions $t \in$ $(-\infty, T] \mapsto\|\varphi(t)\|_{L^{2}\left(\omega_{j}\right)}^{2}$ for $j=1,2$ it follows that if $I$ is of positive measure, then $I=(-\infty, T]$.

We now employ the Fourier expansion of the solution $\varphi$. We have

$$
\varphi(x, t)=\sum_{k \geq 1} \beta_{k} e^{\lambda_{k}(t-T)} w_{k}(x),
$$

where $\left\{\lambda_{k}\right\}_{k \geq 1}$ are the eigenvalues of the Dirichlet Laplacian and $\left\{w_{k}\right\}_{k \geq 1}$ is an orthogonal basis of $L^{2}(\Omega)$ consisting of the corresponding eigenfunctions. 
We also have

$\int_{\omega_{j}} \varphi^{2}(x, t) d x=\sum_{k, \ell \geq 1} \beta_{k} \beta_{\ell} e^{\left(\lambda_{k}+\lambda_{\ell}\right)(t-T)} \gamma_{k, \ell}^{j} \quad$ where $\quad \gamma_{k, \ell}^{j}=\int_{\omega_{j}} w_{k}(x) w_{\ell}(x) d x$.

Let us now assume that $I_{+}=(-\infty, T]$. Then

$$
\sum_{k, \ell \geq 1} \beta_{k} \beta_{\ell} e^{\left(\lambda_{k}+\lambda_{\ell}\right)(t-T)}\left[\gamma_{k, \ell}^{1}-\gamma_{k, \ell}^{2}\right]=0, \quad \forall t \leq T
$$

Multiplying this expression by $\exp (\Lambda t)$ and passing to the limit we deduce that

$$
\sum_{\lambda_{k}+\lambda_{\ell}=\Lambda} \beta_{k} \beta_{\ell}\left[\gamma_{k, \ell}^{1}+\gamma_{k, \ell}^{2}\right]=0
$$

for all $\Lambda>0$.

Let us assume that

$\left(H_{1}\right)$ for every $\Lambda>0$, there is only one pair $\left(\lambda_{k}, \lambda_{\ell}\right)$ such that $\lambda_{k}+\lambda_{\ell}=\Lambda$.

Then

$$
\sum_{k, \ell \geq 1} \beta_{k} \beta_{\ell} e^{\left(\lambda_{k}+\lambda_{\ell}\right)(t-T)}\left[\gamma_{k, \ell}^{1}-\gamma_{k, \ell}^{2}\right]=0, \quad \forall t \leq T
$$

Arguing as above we deduce that

$$
\sum_{\lambda_{k}+\lambda_{\ell}=\Lambda} \beta_{k} \beta_{\ell}\left[\gamma_{k, \ell}^{1}-\gamma_{k, \ell}^{2}\right]=0
$$

Under assumption $\left(H_{1}\right)$ we have, in particular, $\alpha_{m}^{2}\left[\gamma_{m, m}^{1}-\gamma_{m, m}^{2}\right]=0$.

Obviously, given two arbitrary open subsets $\omega_{1}$ and $\omega_{2}$ of $\Omega$, we cannot exclude that, for some value of $m \geq 1, \gamma_{m, m}^{1}-\gamma_{m, m}^{2}=0$, in which case we would not be able to conclude that $\alpha_{m}=0$. But, by making, if necessary, an arbitrarily small deformation of one of the domains one can arrange that $\gamma_{m, m}^{1}-\gamma_{m, m}^{2} \neq 0$. In fact, using the classical tools developed for proving the generic simplicity of the spectrum of the Laplacian (see [17], [14]), one can easily show that, generically with respect to the subsets $\omega_{1}$ and $\omega_{2}$ of the domain $\Omega, \gamma_{m, m}^{1}-\gamma_{m, m}^{2} \neq 0$ for all $m \geq 1$.

This shows that, under the assumption $\left(H_{1}\right)$, generically with respect to the subdomains $\omega_{1}$ and $\omega_{2}$, the switching property is fulfilled. More precisely, we see that under assumption $\left(H_{1}\right)$ and

$$
\int_{\omega_{1}} w_{m}^{2} d x \neq \int_{\omega_{2}} w_{m}^{2} d x, \quad \forall m \geq 1
$$

it follows that the equation 6.1 is null controllable in $L^{2}(\Omega)$ in an arbitrarily small time $T>0$ with switching controls satisfying 6.3.

\footnotetext{
4 Note that this is the infinite-dimensional analogue of the assumption $(H)$ introduced in Section 2.2 when discussing vector valued controls.
} 
On the other hand, according to the results in [15], generically with respect to the domain $\Omega$, the assumption $\left(H_{1}\right)$ is satisfied within the class of domains which are topological balls.

Summarizing, we see that the switching strategy we propose here works generically with respect to the domain $\Omega$ and the subdomains $\omega_{1}$ and $\omega_{2}$.

As in Section 3.1, when these conditions fail the controls are of relaxed switching form.

\subsection{Boundary controls}

The arguments of the previous section apply in the context of boundary control. The problem can now be formulated for the following controlled system:

$$
\begin{cases}y_{t}-\Delta y=0 & \text { in } Q, \\ y=u_{1}(x, t) 1_{\Gamma_{1}}+u_{2}(x, t) 1_{\Gamma_{2}} & \text { on } \Sigma, \\ y(x, 0)=y^{0}(x) & \text { in } \Omega,\end{cases}
$$

where $\Gamma_{1}$ and $\Gamma_{2}$ are two open non-empty subsets of the boundary $\partial \Omega$. The switching condition can now be written as

$$
\left\|u_{1}(t)\right\|_{L^{2}\left(\Gamma_{1}\right)}\left\|u_{2}(t)\right\|_{L^{2}\left(\Gamma_{2}\right)}=0, \quad \text { a.e. } t \in(0, T) .
$$

The same techniques apply in this case, under the condition $\left(H_{1}\right)$ (which holds generically with respect to the domain $\Omega$ within the class of domains which are topological balls (see [15]) and by replacing $\left(\mathrm{H}_{2}\right)$ by the following boundary version of it:

$$
\int_{\Gamma_{1}}\left|\frac{\partial w_{m}}{\partial v}\right|^{2} d \sigma \neq \int_{\Gamma_{2}}\left|\frac{\partial w_{m}}{\partial v}\right|^{2} d \sigma, \quad \forall m \geq 1 .
$$

In the 1-d case this condition cannot be satisfied, as we have seen in the previous section. For that reason we modified the functional $J_{s}$ by adding a weighting factor $\alpha$. But in the multi-dimensional case, for a given domain $\Omega$, the condition holds generically within the class of open subsets $\Gamma_{1}$ and $\Gamma_{2}$ of the boundary.

As in Section 3.1, when these conditions fail the controls are of relaxed switching form.

\subsection{Generic spectral properties}

In the previous sections we have used in an essential manner the result in [15] showing that the condition $\left(H_{1}\right)$ holds generically with respect to the domain $\Omega$.

The same question may be formulated with respect to variations of the coefficients of the equation.

To be more precise, we can consider eigenvalue problems with variable density

$$
\begin{cases}-\Delta w=\lambda(1+\rho(x)) w, & x \in \Omega, \\ w=0, & x \in \partial \Omega .\end{cases}
$$


The question is then whether arbitrarily small variations of the density $\rho$ may guarantee this equation not to have any non-trivial solution.

This problem can be addressed with the classical tools allowing one to prove the generic simplicity of the spectrum. Computing the derivative of the eigenvalues with respect to $\rho$ at $\rho=0$ we get

$$
\lambda^{\prime}=\int_{\Omega} \rho(x) w^{2}(x) d x .
$$

Therefore, if the domain $\Omega$ is, exceptionally, such that the equation

$$
\lambda_{k}+\lambda_{\ell}=\Lambda
$$

has two different pairs of solutions $\left(\lambda_{k}, \lambda_{\ell}\right)$ and $\left(\lambda_{p}, \lambda_{q}\right)$, then the equality

$$
\lambda_{k}+\lambda_{\ell}=\lambda_{p}+\lambda_{q}
$$

holds. In case the derivatives of the two sides of the identity are different in some direction $\rho=\rho(x)$, arbitrarily small deformations of the density coefficient in that direction would immediately make the identity 6.14) fail. In view of the expression of the derivative this would require that

$$
\int_{\Omega} \rho(x)\left[w_{k}^{2}+w_{\ell}^{2}\right] d x \neq \int_{\Omega} \rho(x)\left[w_{p}^{2}+w_{q}^{2}\right] d x .
$$

This holds if the squares of the eigenfunctions are linearly independent, a result which has recently been proved in [15] generically with respect to the domain $\Omega$ within the class of domains which are topological balls.

The same question can be formulated for variations of the density localized in some subset $\mathcal{O}$ of the domain $\Omega$. To do this one needs to know whether the squares of the eigenfunctions are linearly independent when restricted to $\mathcal{O}$. Using the analyticity of eigenfunctions this can be shown to hold for all open non-empty subsets $\mathcal{O}$ (and even for any measurable set of positive measure) if it holds in the domain $\Omega$, a property that is known to be generically true in the class of topological balls (see [15]).

Note that the same problem was formulated in [8] in connection with the optimal design of dampers for dissipative wave equations. Similar issues arise for 1-d SturmLiouville problems. In that context, it is well known that there are many counterexamples to this linear independence property (see [10]). But this is not incompatible with the property being true generically.

\section{Further comments and open problems}

The results of this paper can be extended in various ways.

Approximate controllability. The same methods can be applied in the context of approximate controllability or finite-approximate controllability. For instance, in the context of approximate controllability of the heat equation (6.1) in which the goal is to drive the 
solution from an initial datum $y^{0}$ to an $\epsilon$-neighborhood of a final target $y^{1}$, it is sufficient to modify the functional (6.4) to the following one:

$$
\begin{aligned}
J_{a p s}\left(\varphi^{0}\right)= & \frac{1}{2} \int_{0}^{T} \max \left(\int_{\omega_{1}}|\varphi(x, t)|^{2} d x, \int_{\omega_{2}}|\varphi(x, t)|^{2} d x\right) d t \\
& +\epsilon\left\|\varphi^{0}\right\|_{L^{2}(\Omega)}-\int_{0}^{1} y^{0}(x) \varphi(x, 0) d x+\int_{0}^{1} y^{1}(x) \varphi^{0}(x) d x .
\end{aligned}
$$

The rest of the analysis remains unchanged. The conclusions are also the same: under the same spectral conditions $\left(H_{1}\right)$ and the same assumption $\left(H_{2}\right)$ on the subdomains $\omega_{1}$ and $\omega_{2}$, the controls obtained by minimizing this functional are of switching form.

There are also several issues, worth addressing, that have not been considered in this paper. We mention here some of them.

Complexity of the switching structure. It would be natural to address the issue of the number of switchings that the controls obtained through the variational principle introduced in this article perform. This is a completely open issue.

More general parabolic systems. All our developments in the context of the heat equation are based on Fourier series expansions. It would be interesting to see if the methodology we have applied and the results on switching controls can be adapted to more general equations involving, for instance, potentials depending both on space and time. There is a rich literature on the null control of those equations (see [19]). But the problem of switching controls has not been addressed so far. Obviously, the main issue to address in this case is how to show that, under suitable geometric conditions on the domains $\Omega, \omega_{1}$ and $\omega_{2}$, the fact that the measure of the set of time instants $t \in(0, T)$ such that

$$
\|\hat{\varphi}(t)\|_{L^{2}\left(\omega_{2}\right)}=\|\hat{\varphi}(t)\|_{L^{2}\left(\omega_{1}\right)}
$$

vanishes whenever $\varphi$ is a non-trivial solution of a heat equation with potential of the form

$$
\begin{cases}\varphi_{t}+\Delta \varphi+p(x, t) \varphi=0 & \text { in } Q, \\ \varphi=0 & \text { on } \Sigma, \\ \varphi(x, T)=\varphi^{0}(x) & \text { in } \Omega,\end{cases}
$$

$p$ being a measurable and bounded potential, for instance, implies that $\varphi$ is identically equal to zero.

The same can be said in the context of the pointwise control of the 1-d heat equation with time-dependent potentials.

The wave equation. The same problems make sense for the wave equation. Consider for instance the 1-d case with two pointwise controllers:

$$
\begin{cases}y_{t t}-y_{x x}=u_{a}(t) \delta_{a}+u_{b}(t) \delta_{b}, & 0<x<1,0<t<T, \\ y(0, t)=y(1, t)=0, & 0<t<T \\ y(x, 0)=y^{0}(x), \quad y_{t}(x, 0)=y^{1}(x), & 0<x<1 .\end{cases}
$$


Consider also the corresponding adjoint system

$$
\begin{cases}\varphi_{t t}-\varphi_{x x}=0, & 0<x<1,0<t<T, \\ \varphi(0, t)=\varphi(1, t)=0, & 0<t<T, \\ \varphi(x, T)=\varphi^{0}(x), \quad \varphi_{t}(x, T)=\varphi^{1}(x), & 0<x<1 .\end{cases}
$$

The key issue to address to show that the methods above apply and yield switching controls is whether the sets

$$
I_{ \pm}=\{t \in(0, T): \varphi(a, t) \pm \varphi(b, t)=0\}
$$

can be guaranteed to be of null measure when $\varphi$ is a non-trivial solution of the adjoint system. Obviously, due to the finite velocity of propagation, this is far from being the case. Indeed, it is easy to build non-trivial solutions of the adjoint wave equation that for a short time interval $(0, \tau)$ satisfy, for instance,

$$
\varphi(a, t)=\varphi(b, t), \quad \forall t \in(0, \tau) .
$$

In particular, the arguments we have used above for the heat equation do not apply in the present setting because of the lack of time analyticity of solutions.

It would be interesting to develop new methods allowing one to build switching controllers for wave like equations or, more generally, in the absence of time analyticity of solutions of the semigroups under consideration.

The 1-d wave equation has been discussed in [5] where sufficient conditions for the existence of switching controls are given by means of the d'Alembert representation formula. This issue is also related to the on-off stabilization property analyzed in [11].

Acknowledgments. The author acknowledges fruitful comments of José M. Arrieta, S. Ervedoza, M. Gugat, T. Seidman and M. Sigalotti. He also thanks A. Marica for the plots in Section 2.3

This work was partially supported by the Grant MTM2008-03541 of the MICINN, Spain, the ERC Advanced Grant FP7-246775 NUMERIWAVES and Grant PI2010-04 of the Basque Government.

\section{References}

[1] Castro, C., Zuazua, E.: Unique continuation and control for the heat equation from an oscillating lower dimensional manifold. SIAM J. Control Optim. 43, 1400-1434 (2005) Zbl 1101.93010 MR 2124279

[2] Dager, R., Zuazua, E.: Wave Propagation and Control in 1-d Vibrating Multi-Structures. Math. Appl. 50, Springer (2006) Zbl 1083.74002 MR 2169126

[3] Fabre, C., Puel, J., Zuazua, E.: Approximate controllability for the semilinear heat equation. Proc. Roy. Soc. Edinburgh Sect. A 125, 31-61 (1995)

[4] Gantmacher, F. R.: The Theory of Matrices, Vols. I and II. Chelsea, New York (1959) Zbl 0927.15001 Zbl 0927.15002 MR 0107649

[5] Gugat, M.: Optimal switching boundary control of a string to rest in finite time. Z. Angew. Math. Mech. 48, 283-305 (2008) Zbl pre05271062 MR 2389094 
[6] Gugat, M., Leugering, G.: $L^{\infty}$-Norm minimal control of the wave equation: on the weakness of the bang-bang principle. ESAIM Control Optim. Calc. Var. 14, 254-283 (2008) Zbl 1133.49006 MR 2394510

[7] Hante, F., Leugering, G., Seidman, T.: Modeling and analysis of modal switching in networked transport systems, Appl. Math. Optim. 59, 275-292 (2009). Zbl 1167.93005 MR 2480783

[8] Hébrard, P., Henrot, A.: A spillover phenomenon in the optimal location of actuators. SIAM J. Control Optim. 44, 349-366 (2005) Zbl 1083.49002 MR 2177160

[9] Lee, E. B., Markus, L.: Foundations of Optimal Control Theory, SIAM Ser. Appl. Math., Wiley (1967). Zbl 0159.13201 MR 0220537

[10] Mahar, T. J., Willner, B. E.: Sturm-Liouville eigenvalue problems in which the squares of the eigenfunctions are linearly dependent, Comm. Pure Appl. Math. 33, 567-578 (1980) Zbl 0422.34024 MR 0575738

[11] Martinez, P., Vancostenoble, J.: Stabilization of the wave equation by on-off and positivenegative feedbacks, ESAIM Control Optim. Calc. Var. 7, 335-377 (2002) Zbl 1026.35061 MR 1925033

[12] Micu, S., Zuazua, E.: On the controllability of a fractional order parabolic equation. SIAM J. Control Optim. 44, 1950-1972 (2006) Zbl 1116.93022 MR 2248170

[13] Micu, S., Zuazua, E.: An introduction to the controllability of linear PDE. In: Contrôle non linéaire et applications, T. Sari (ed.), Travaux en Cours, Hermann, 67-150 (2005) Zbl pre05046349

[14] Ortega, J., Zuazua, E.: Generic simplicity of the spectrum and stabilization for a plate equation. SIAM J. Control Optim. 39, 1585-1614 (2001); Addendum: SIAM J. Control Optim. 42, 1905-1910 (2004) Zbl 0980.35118 MR 1825594 MR 2046392

[15] Privat, Y., Sigalotti, M.: The squares of the Laplacian-Dirichlet eigenfunctions are generically linearly independent. ESAIM Control Optim. Calc. Var. 16, 794-805, 806-807 (2010) Zbl pre05768973

[16] Shorten, R., Wirth, F., Mason, O., Wulff, K., King, Ch.: Stability criteria for switched and hybrid systems. SIAM Rev. 49, 545-592 (2007) Zbl 1127.93005 MR 2375524

[17] Uhlenbeck, K.: Generic properties of eigenfunctions. Amer. J. Math. 98, 1059-1078 (1976) Zbl 0355.58017 MR 0464332

[18] Zuazua, E.: Propagation, observation, and control of waves approximated by finite difference methods. SIAM Rev. 47, 197-243 (2005) Zbl 1077.65095 MR 2179896

[19] Zuazua, E.: Controllability and observability of partial differential equations: Some results and open problems. In: Handbook of Differential Equations: Evolutionary Equations, Vol. 3, C. M. Dafermos and E. Feireisl (eds.), Elsevier, 527-621 (2006) MR 2549374 